\title{
WestVirginiaUniversity
}

THE RESEARCH REPOSITORY @ WVU

Graduate Theses, Dissertations, and Problem Reports

2003

\section{Eulerian subgraphs and Hamiltonicity of claw -free graphs}

Mingquan Zhan

West Virginia University

Follow this and additional works at: https://researchrepository.wvu.edu/etd

\section{Recommended Citation}

Zhan, Mingquan, "Eulerian subgraphs and Hamiltonicity of claw -free graphs" (2003). Graduate Theses, Dissertations, and Problem Reports. 1910.

https://researchrepository.wvu.edu/etd/1910

This Dissertation is protected by copyright and/or related rights. It has been brought to you by the The Research Repository @ WVU with permission from the rights-holder(s). You are free to use this Dissertation in any way that is permitted by the copyright and related rights legislation that applies to your use. For other uses you must obtain permission from the rights-holder(s) directly, unless additional rights are indicated by a Creative Commons license in the record and/ or on the work itself. This Dissertation has been accepted for inclusion in WVU Graduate Theses, Dissertations, and Problem Reports collection by an authorized administrator of The Research Repository @ WVU.

For more information, please contact researchrepository@mail.wvu.edu. 


\title{
Eulerian Subgraphs and Hamiltonicity of Claw-free Graphs
}

\author{
Mingquan Zhan \\ Dissertation submitted to the \\ Eberly College of Arts and Sciences \\ at West Virginia University \\ in partial fulfillment of the requirements \\ for the degree of \\ Doctor of Philosophy \\ in \\ Mathematics \\ Hong-Jian Lai, Ph.D., Chair \\ Elaine M Eschen, Ph.D. \\ John Goldwasser, Ph.D. \\ Jerzy Wojcienchowski, Ph.D. \\ Cun-Quan Zhang, Ph.D. \\ Department of Mathematics \\ Morgantown, West Virginia \\ 2003
}

Keywords: Supereulerian, Line graph, Claw-free graph, Hamiltonicity, Collapsible, Reduction, Closure

Copyright 2003 Mingquan Zhan 


\section{ABSTRACT \\ Eulerian Subgraphs and Hamiltonicity of Claw-free Graphs}

\section{Mingquan Zhan}

Let $C(l, k)$ denote the class of 2-edge-connected graphs of order $n$ such that a graph $G \in C(l, k)$ if and only if for every edge cut $S \subseteq E(G)$ with $|S| \leq 3$, each component of $G-S$ has order at least $\frac{n-k}{l}$. We prove that If $G \in C(6,0)$, then $G$ is supereulerian if and only if $G$ cannot be contracted to $K_{2,3}, K_{2,5}$ or $K_{2,3}(e)$, where $e \in E\left(K_{2,3}\right)$ and $K_{2,3}(e)$ stands for a graph obtained from $K_{2,3}$ by replacing $e$ by a path of length 2 . Previous results by Catlin and Li, and by Broersma and Xiong are extended.

We also investigate the supereulerian graph problems within planar graphs, and we prove that if a 2-edge-connected planar graph $G$ is at most three edges short of having two edge-disjoint spanning trees, then $G$ is supereulerian except a few classes of graphs. This is applied to show the existence of spanning Eulerian subgraphs in planar graphs with small edge cut conditions. We determine several extremal bounds for planar graphs to be supereulerian.

Kuipers and Veldman conjectured that any 3-connected claw-free graph with order $n$ and minimum degree $\delta \geq \frac{n+6}{10}$ is Hamiltonian for $n$ sufficiently large. We prove that if $H$ is a 3-connected claw-free graph with sufficiently large order $n$, and if $\delta(H) \geq \frac{n+5}{10}$, then either $H$ is hamiltonian, or $\delta(H)=\frac{n+5}{10}$ and the Ryjácek's closure $c l(H)$ of $H$ is the line graph of a graph obtained from the Petersen graph $P_{10}$ by adding $\frac{n-15}{10}$ pendant edges at each vertex of $P_{10}$. 


\section{Acknowledgements}

I would like to thank my supervisor, Dr. Hong-Jian Lai, for his guidance, advice, and continuing encouragement. It is a pleasure to work under his supervision. Without him, this paper could not have come about.

I would also like to thank my other committee members: Dr. Elaine Eschen, Dr. John Goldwasser, Dr. Jerzy Wojcienchowski, and Dr. Cun-Quan Zhang, for their help during my studies. I would like to express my gratitude to my English tutor Mrs. Cathy Barger for her help during these two years.

And finally, I thank my family: my wife, Zhongcui, for her love and her never ending support; my parents Jianzhong and Meihua, for their constant encouragement, and my daughter Shuxin, who is my future, for providing me with the gift of the joy of her youth on my most difficult days. 


\section{DEDICATION}

To

my parents

and

my wife 


\section{Contents}

1 Definitions and Notations 1

2 Catlin's Reduction Method 4

3 Eulerian subgraphs and Hamilton-connected line graphs 15

3.1 Introduction . . . . . . . . . . . . . . . . . 15

3.2 Proofs of Theorems 3.1 .3 and $3.1 .5 \ldots \ldots \ldots \ldots$

3.3 Applications . . . . . . . . . . . . . . . . . . . . . . . . . 19

4 Supereulerian planar graphs $\quad 21$

4.1 Introduction . . . . . . . . . . . . . . . . . 21

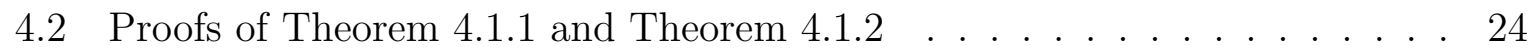

4.3 Proofs of Theorems 4.1 .3 and $4.1 .4 \ldots \ldots \ldots$

4.4 Proofs of Theorems 4.1 .7 and $4.1 .8 \ldots \ldots \ldots$. . . . . . . . . . . . . 34

4.5 Proofs of Theorems 4.1 .9 and $4.1 .10 \ldots \ldots \ldots \ldots$ 
5 Hamiltonicity in 3-connected claw-free graphs

5.1 Introduction . . . . . . . . . . . . . . . . . . . . 39

5.2 Proof of Theorem 5.1.5 . . . . . . . . . . . . . . . . . . 40 


\section{Chapter 1}

\section{Definitions and Notations}

We use [1] for terminology and notations not defined here and consider finite, undirected graphs. We allow graphs to have multiple edges but not loops. A graph with at least two vertices is called a nontrivial graph.

Let $G$ be a graph. We denote an $n$-cycle by $C_{n}$. We use $\kappa(G), \kappa^{\prime}(G)$ to denote the connectivity and the edge-connectivity of $G$, respectively. The degree and neighborhood of a vertex $x$ of $G$ are respectively denoted by $d_{G}(x)$ and $N_{G}(x)$, and the minimum degree of $G$ is denoted by $\delta(G)$. An edge $e=u v$ is called a pendant edge if either $d_{G}(u)=1$ or $d_{G}(v)=1$. If $S \subseteq V(G)$ and $X_{1} \subseteq E(G), G[S]$ and $G\left[X_{1}\right]$ are the subgraphs induced in $G$ by $S$ and $X_{1}$, respectively. A vertex $v \in G$ is called a locally connected vertex if $G\left[N_{G}(v)\right]$ is connected. For $H \subseteq G, x \in V(G)$ and $A, B \subseteq V(G)$ with $A \cap B=\emptyset$, let $N_{H}(x)=N_{G}(x) \cap V(H), d_{H}(x)=\left|N_{H}(x)\right|, N_{H}(A)=\bigcup_{v \in A} N_{H}(v)$, $[A, B]_{G}=\{u v \in E(G) \mid u \in A, v \in B\}$, and $G-A=G[V(G)-A]$. When $A=\{v\}$, we use $G-v$ for $G-\{v\}$. If $H \subseteq G$, then for an edge subset $X \subseteq E(G)-E(H)$, we write $H+X$ for $G[E(H) \cup X]$.

A graph $G$ is essentially $k$-edge-connected if $|E(G)| \geq k+1$ and if for every $E_{0} \subseteq E(G)$ with $\left|E_{0}\right|<k, G-E_{0}$ has exactly one component $H$ with $E(H) \neq \emptyset$. The greatest integer $k$ such that $G$ is essentially $k$-edge-connected is the essential edgeconnectivity $\kappa_{e}(G)$ of $G$. For each $i=0,1,2, \cdots$, let $D_{i}(G)=\left\{v \in V(G) \mid d_{G}(v)=i\right\}$, 
and for any integer $t \geq 1$, let $D_{t}^{*}(G)=\bigcup_{i \geq t} D_{i}(G)$. The edge arboricity $a_{1}(G)$ of $G$ is the minimum number of edge-disjoint forests whose union equals $G$. The girth of $G$, denoted by $g(G)$, is the length of a shortest cycle of $G$, or $\infty$ if $G$ is acyclic.

Let $O(G)$ denote the set of odd degree vertices of $G$. A graph $G$ is Eulerian if $O(G)=\emptyset$ and $G$ is connected. A graph $G$ is supereulerian if $G$ has a spanning Eulerian subgraph. In particular, $K_{1}$ is both eulerian and supereulerian. Denote by $\mathcal{S} \mathcal{L}$ the family of all supereulerian graphs.

Let $X \subseteq E(G)$. The contraction $G / X$ is the graph obtained from $G$ by identifying the two ends of each edge in $X$ and then deleting the resulting loops. We define $G / \emptyset=G$. If $K$ is a subgraph of $G$, then we write $G / K$ for $G / E(K)$. Note that even if $G$ is a simple graph, contracting some edges of $G$ may result in a graph with multiple edges. If $K$ is a connected subgraph of $G$, and if $v_{K}$ is the vertex in $G / K$ onto which $K$ is contracted, then $K$ is called the preimage of $v_{K}$, and is denoted by $P I\left(v_{K}\right)$. A vertex $v$ in a contraction of $G$ is nontrivial if $P I(v)$ has at least one edge.

The line graph of a graph $G$, denote by $L(G)$, has $E(G)$ as its vertex set, where two vertices in $L(G)$ are adjacent if and only if the corresponding edges in $G$ are adjacent.

A subgraph $H$ of a graph $G$ is dominating if $G-V(H)$ is edgeless. Let $v_{0}, v_{k} \in V(G)$. A $\left(\mathbf{v}_{\mathbf{0}}, \mathbf{v}_{\mathbf{k}}\right)$-trail of $G$ is a vertex-edge alternating sequence

$$
v_{0}, e_{1}, v_{1}, e_{2}, \cdots, e_{k}, v_{k}
$$

such that all the $e_{i}$ 's are distinct and such that for each $i=1,2, \cdots, k, e_{i}$ is incident with both $v_{i-1}$ and $v_{i}$. With the notation above, this $\left(v_{0}, v_{k}\right)$-trail is also called an $\left(\mathbf{e}_{\mathbf{1}}, \mathbf{e}_{\mathbf{k}}\right)$ trail. All the vertices in $v_{1}, v_{2}, \cdots, v_{k-1}$ are internal vertices of trail. A dominating $\left(\mathbf{e}_{\mathbf{1}}, \mathbf{e}_{\mathbf{k}}\right)$-trail $T$ of $G$ is an $\left(e_{1}, e_{k}\right)$-trail such that every edge of $G$ is incident with an internal vertex of $T$. A spanning $\left(\mathbf{e}_{\mathbf{1}}, \mathbf{e}_{\mathbf{k}}\right)$-trail of $G$ is a dominating $\left(e_{1}, e_{k}\right)$-trail such that $V(T)=V(G)$. There is a close relationship between dominating eulerian subgraphs in graphs and Hamilton cycles in $L(G)$.

Theorem 1.0.1 (Harary and Nash-Williams, [16]) Let $G$ be a graph with $|E(G)| \geq 3$. Then $L(G)$ is hamiltonian if and only if $G$ has a dominating eulerian subgraph. 
A graph $G$ is Hamilton-connected if for each $u, v \in V(G)(u \neq v)$, there exists a $(u, v)$-path containing all vertices of $G$. With a similar argument in the proof of Theorem 1.0.1, one can obtain a theorem for Hamilton-connected line graphs.

Theorem 1.0.2 Let $G$ be a graph with $|E(G)| \geq 3$. Then $L(G)$ is Hamilton-connected if and only if for any pair of edges $e_{1}, e_{2} \in E(G), G$ has a dominating $\left(e_{1}, e_{2}\right)$-trail.

We say that an edge $e \in E(G)$ is subdivided when it is replaced by a path of length 2 whose internal vertex, $v(e)$, has degree 2 in the resulting graph. The process of taking an edge $e$ and replacing it by the length 2 path is called subdividing e. For a graph $G$ and edges $e_{1}, e_{2} \in E(G)$, let $G\left(e_{1}\right)$ denote the graph obtained from $G$ by subdividing $e_{1}$, and let $G\left(e_{1}, e_{2}\right)$ denote the graph obtained from $G$ by subdividing both $e_{1}$ and $e_{2}$. Thus

$$
V\left(G\left(e_{1}, e_{2}\right)\right)-V(G)=\left\{v\left(e_{1}\right), v\left(e_{2}\right)\right\} .
$$

From the definitions, one immediately has the following observation.

Proposition 1.0.3 Let $G$ be a graph and $e_{1}, e_{2} \in E(G)$. If $G\left(e_{1}, e_{2}\right)$ has a spanning $\left(v\left(e_{1}\right), v\left(e_{2}\right)\right)$-trail, then $G$ has a spanning $\left(e_{1}, e_{2}\right)$-trail. 


\section{Chapter 2}

\section{Catlin's Reduction Method}

In [4] Catlin defined collapsible graphs. For $R \subseteq V(G)$, a subgraph $\Gamma$ of $G$ is called an $\mathbf{R}$-subgraph if both $O(\Gamma)=R$ and $G-E(\Gamma)$ is connected. A graph is collapsible if $G$ has an $R$-subgraph for every even set $R \subseteq V(G)$. In particular, $K_{1}$ is collapsible. Let $\mathcal{C} \mathcal{L}$ denote the family of all collapsible graphs. For a graph $G$ and its connected subgraph $H, G / H$ denotes the graph obtained from $G$ by contracting $H$, i.e. by replacing $H$ by a vertex $v_{H}$ such that the numbers of edges in $G / H$ joining any $v \in V(G)-V(H)$ to $v_{H}$ in $G / H$ equals the number of edges joining $v$ in $G$ to $H$. A graph $G$ is contractible to a graph $G^{\prime}$ if $G$ contains pairwise vertex-disjoint connected subgraphs $H_{1}, H_{2}, \cdots, H_{k}$ with $\bigcup_{i=1}^{k} V\left(H_{i}\right)=V(G)$ such that $G^{\prime}$ is obtained from $G$ by successively contracting $H_{1}, H_{2}$, $\cdots, H_{k}$. The subgraph $H_{i}$ of $G$ is called the pre-image of the vertex $v_{H_{i}}$ of $G^{\prime}$. Catlin [4] showed that every graph $G$ has a unique collection of pairwise vertex-disjoint maximal collapsible subgraphs $H_{1}, H_{2}, \cdots, H_{k}$ such that $\bigcup_{i=1}^{k} V\left(H_{i}\right)=V(G)$. The reduction of $G$ is the graph obtained from $G$ by successively contracting $H_{1}, H_{2}, \cdots, H_{k}$. A graph is reduced if it is the reduction of some graph. A nontrivial vertex in $G^{\prime}$ is a vertex that is the contraction image of a nontrivial connected subgraph of $G$. Note that if $G$ has an $O(G)$-subgraph $\Gamma$, then $G-E(\Gamma)$ is a spanning Eulerian subgraph of $G$. Therefore, every collapsible graph is supereulerian. 
Theorem 2.0.4 (Catlin, [4]) Let $G$ be a connected graph.

(i) If $G$ has a spanning tree $T$ such that each edge of $T$ is in a collapsible subgraph of $G$, then $G$ is collapsible.

(ii) If $G$ is reduced, then $G$ is a simple graph and has no cycle of length less than four.

(iii) $G$ is reduced if and only if $G$ has no nontrivial collapsible subgraphs.

(iv) Let $G^{\prime}$ be the reduction of $G$. Then $G \in \mathcal{S L}$ if and only if $G^{\prime} \in \mathcal{S L}$, and $G \in \mathcal{C} \mathcal{L}$ if and only if $G^{\prime}=K_{1}$.

Jaeger in [17] showed that if $G$ has two edge-disjoint spanning trees, then $G$ is supereulerian. Letting $F(G)$ be the minimum number of additional edges that must be added to $G$ so that the resulting graph has two edge-disjoint spanning trees, Catlin [4] and Catlin et al. [8] improved Jaeger's result. We combine these results in the following theorem.

Theorem 2.0.5 Let $G$ be a graph. Then each of the following holds.

(i)(Jaeger, [17]) If $F(G)=0$, then $G$ is supereulerian.

(ii)(Catlin, [4]) If $F(G) \leq 1$ and if $G$ is connected, then $G$ is collapsible if and only if $G$ is not contractible to a $K_{2}$.

(iii)(Catlin, Han and Lai, [8]) If $F(G) \leq 2$ and if $G$ is connected, then either $G$ is collapsible, or the reduction of $G$ is a $K_{2}$ or a $K_{2, t}$ for some integer $t \geq 1$.

Theorem 2.0.6 (Catlin, Han and Lai, [8]) Let $G$ be a connected reduced graph. If $F(G) \leq 2$, then exactly one of following holds:

(i) $G \in \mathcal{S L}$;

(ii) $G$ has a cut edge;

(iii) $G$ is $K_{2, s}$ for some odd integer $s \geq 3$.

In 1987, Catlin [6] introduced a reduction technique of the 4-cycle. Let $G$ be a graph and let $C=v_{1} v_{2} v_{3} v_{4} v_{1}$ be a 4 -cycle of $G$. Let $G_{\pi}$ denote the graph obtained from $G-E(C)$ by identifying $v_{1}$ and $v_{3}$ to form a single vertex $w_{1}$, and by identifying $v_{2}$ and $v_{4}$ to form a single vertex $w_{2}$, and by joining $w_{1}$ and $w_{2}$ with a new edge $e_{\pi}$. 
Theorem 2.0.7 Each of the following holds:

(i) (Catlin, [6]) If $G_{\pi}$ is collapsible, then $G$ is collapsible; if $G_{\pi}$ is supereulerian, then $G$ is supereulerian.

(ii) (Catlin, Han and Lai, [8]) If $G$ is reduced, then $F\left(G_{\pi}\right)=F(G)-1$.

(iii) (Catlin, [6]) Let $K_{3,3}-e$ denote the graph obtained from $K_{3,3}$ by removing an edge. Then $K_{3,3}-e, K_{n}(n \geq 3)$ and $C_{2}$ are collapsible.

(iv) (Catlin, [6]) If $G$ is reduced, then $a_{1}(G) \leq 2$; if $a_{1}(G) \leq 2$, then $F(G)=2|V(G)|-$ $|E(G)|-2$.

Applying Theorem 2.0.7(i), we have the following lemma.

Lemma 2.0.8 Let $G_{1}, G_{2}, G_{3}$ be the graphs given in Figure 2.1. Then $G_{1}, G_{2}$ and $G_{3}$ are collapsible.

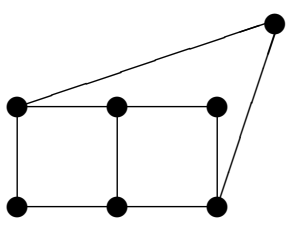

$G_{1}$

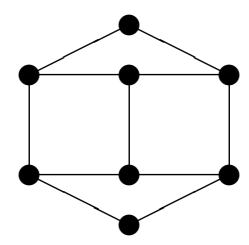

$G_{2}$

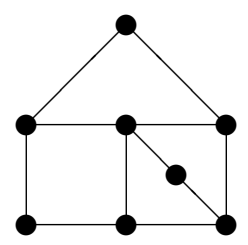

$G_{3}$

Figure 2.1

Let $t, s_{1}, s_{2}, s_{3}$ be nonnegative integers. Denote $K_{2, t}^{\prime}, K_{2, t}^{\prime \prime}, K_{1,3}\left(s_{1}, s_{2}, s_{3}\right), S\left(s_{1}, s_{2}\right)$, $J\left(s_{1}, s_{2}\right), J^{\prime}\left(s_{1}, s_{2}\right)$ to be graphs shown in Figure 2.2, where the $s_{i}(i=1,2,3)$ vertices and the two vertices connected by the two lines shown in each of the graphs forms a $K_{2, s_{i}}$ graph.

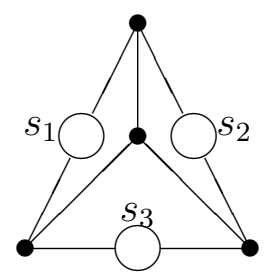

$K_{1,3}\left(s_{1}, s_{2}, s_{3}\right)$

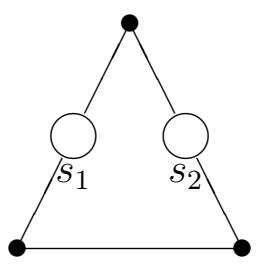

$S\left(s_{1}, s_{2}\right)$

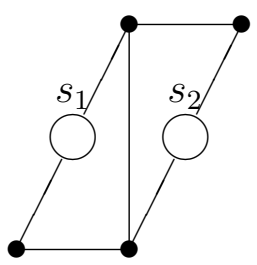

$J\left(s_{1}, s_{2}\right)$

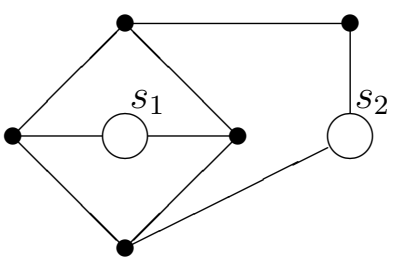

$J^{\prime}\left(s_{1}, s_{2}\right)$ 


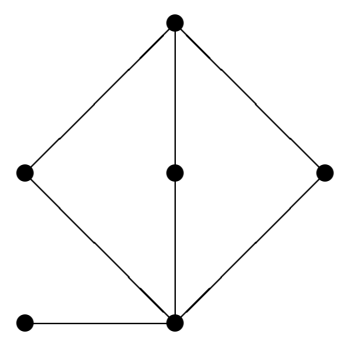

$K_{2, t}^{\prime}$

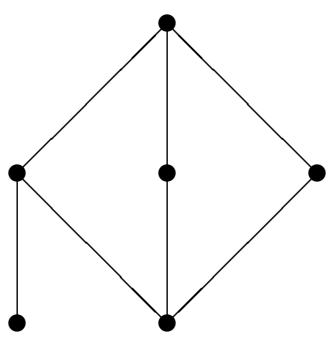

$K_{2, t}^{\prime \prime}$

Figure 2.2

Let

$$
\mathcal{F}=\left\{K_{1}, K_{2}, K_{2, t}, K_{2, t}^{\prime}, K_{2, t}^{\prime \prime}, K_{1,3}\left(s_{1}, s_{2}, s_{3}\right), S\left(s_{1}, s_{2}\right), J\left(s_{1}, s_{2}\right), J^{\prime}\left(s_{1}, s_{2}\right), P\right\},
$$

where $P$ denotes the Petersen graph.

Theorem 2.0.9 (Chen and Lai, [12]) If $G$ is connected reduced graph with $|V(G)| \leq 11$ and $F(G) \leq 3$, then $G \in \mathcal{F}$.

Theorem 2.0.10 (Chen, [10]) Let $G$ be a reduced graph with $n \leq 11$ vertices, and $\kappa^{\prime}(G) \geq 3$. Then $G$ is either $K_{1}$ or the Petersen graph.

Lemma 2.0.11 Let $G$ be a connected simple graph with $n \leq 8$ vertices and with $D_{1}(G)=$ $\emptyset,\left|D_{2}(G)\right| \leq 2$. Then either $G$ is one of three graphs in Figure 2.3, or the reduction of $G$ is $K_{1}$ or $K_{2}$. 

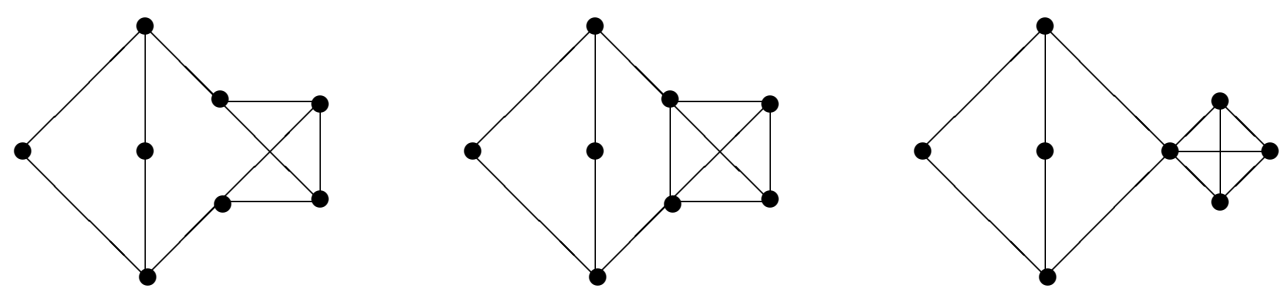

Figure 2.3

Proof. By Theorem 2.0.10, we may assume $\kappa^{\prime}(G) \in\{1,2\}$. Suppose first that $G$ has a cut-edge $e$ and that $G_{1}$ and $G_{2}$ are two components of $G-e$. Let $n_{i}=\left|V\left(G_{i}\right)\right|, 1 \leq i \leq 2$ and assume, without loss of generality, $n_{2} \geq n_{1}$. Since $G$ is simple and $D_{1}(G)=\emptyset, n_{1} \geq 3$ and equality holds if and only if $G_{1}=K_{3}$. If both $n_{2}=n_{1}=4$, then each of $G_{1}$ and $G_{2}$ must be $K_{4}$ or $K_{4}-e$ where $e \in E\left(K_{4}\right)$. Since $K_{3}$ is collapsible and by Theorem 2.0.4(i), both $G_{1}$ and $G_{2}$ are collapsible, and so the reduction of $G$ is $K_{2}$. Since $n_{2}=3$ will force $\left|D_{2}(G)\right| \geq 3$, we assume that $4 \leq n_{2} \leq 5$ and $n_{1}=3$. If $G_{2}$ is not collapsible, then $G_{2} \in\left\{C_{4}, C_{5}, K_{2,3}\right\}$ and $\left|D_{2}(G)\right| \geq 3$. So $G_{2}$ must be collapsible. Hence the reduction of $G$ is $K_{2}$.

Now we assume that $G$ is 2-edge-connected and $G^{\prime}$ is the reduction of $G$ with $n^{\prime}=$ $\left|V\left(G^{\prime}\right)\right| \geq 2$. Then $G^{\prime}$ is 2-edge-connected and nontrivial. Let $C_{m}$ be a longest cycle in $G^{\prime}$. Then $m \geq 4$ by Theorem 2.0.4(ii).

If $n^{\prime}=8$ or 7 , then $G=G^{\prime}$. As $\left|D_{2}(G)\right| \leq 2$, we have $F\left(G^{\prime}\right) \leq 3$ by Theorem 2.0.7(iv). By Theorem 2.0.9 and $\kappa^{\prime}\left(G^{\prime}\right) \geq 2,\left|D_{2}(G)\right| \geq 3$, a contradiction. If $n^{\prime}=6$, then either $G=G^{\prime}$ or the pre-image of a vertex in $G^{\prime}$ is a triangle and the pre-images of the other vertices in $G^{\prime}$ are themselves. Thus $\left|D_{2}\left(G^{\prime}\right)\right| \leq 2$. By Theorem 2.0.7(iv), we have $F\left(G^{\prime}\right) \leq 2$. Therefore $\left|D_{2}\left(G^{\prime}\right)\right| \geq 3$ by Theorem 2.0.5(iii), a contradiction. If $n^{\prime}=4$, then $G^{\prime}=C_{4}$. Note that the size of the pre-image of each vertex is either 1 or at least 3. Thus $\left|D_{2}(G)\right| \geq 3$. It contradicts the hypothesis that $\left|D_{2}(G)\right| \leq 2$. So $n^{\prime}=5$. Note again that the size of the pre-image of each vertex is either 1 or at least 3. By $\left|D_{2}(G)\right| \leq 2,\left|D_{3}\left(G^{\prime}\right)\right| \neq 0$. Thus $F\left(G^{\prime}\right) \leq 2$. By Theorem 2.0.5(iii), $G^{\prime}=K_{2,3}$. As $n \leq 8$ and $\left|D_{2}(G)\right| \leq 2$, the pre-image of a vertex having degree 2 in $G^{\prime}$ is either a $K_{4}$ or a $K_{4}$ minus an edge, and the pre-images of the other vertices in $G^{\prime}$ are themselves. Thus $G$ is 
one of the graphs in Figure 2.3.

Lemma 2.0.12 If $G$ is collapsible, then for any pair of vertices $u, v \in V(G), G$ has a spanning $(u, v)$-trail.

Proof. Let $R=(O(G) \cup\{u, v\})-(O(G) \cap\{u, v\})$. Then $|R|$ is even. Let $\Gamma_{R}$ be an $R$-subgraph of $G$. Then $G-E\left(\Gamma_{R}\right)$ is a spanning $(u, v)$-trail of $G$.

Let $G$ be a graph and let $S \subseteq V(G)$ be a vertex subset. A eulerian subgraph $H$ of $G$ is called an $S$-eulerian subgraph if $S \subseteq V(H)$. Let $K_{2,3}, K_{2,5}, W_{3}^{\prime}, W_{4}^{\prime}, L_{1}, L_{2}$ and $L_{3}$ be the labelled graphs defined in Figures 2.4-2.6, and let $\mathcal{F}=\left\{K_{2,3}, K_{2,5}, W_{3}^{\prime}, W_{4}^{\prime}, L_{1}, L_{2}, L_{3}\right\}$. Using the labels in Figures 2.4-2.6, for each $L \in \mathcal{F}$, we define $B(L)$, the bad set of $L$, to be the vertex subset of $V(L)$ that are labeled with the $b_{i}^{\prime} s$.
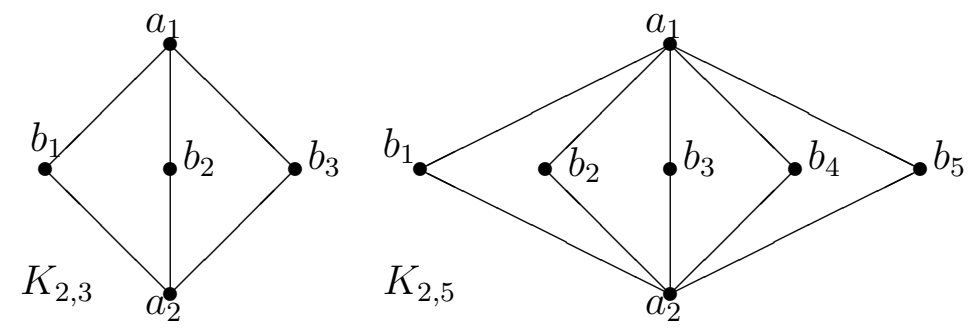

Figure 2.4 The graphs $K_{2,3}$ and $K_{2,5}$
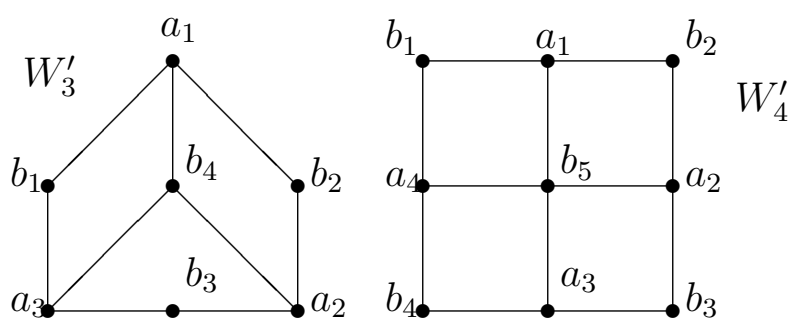

Figure 2.5 The graphs $W_{3}^{\prime}$ and $W_{4}^{\prime}$ 

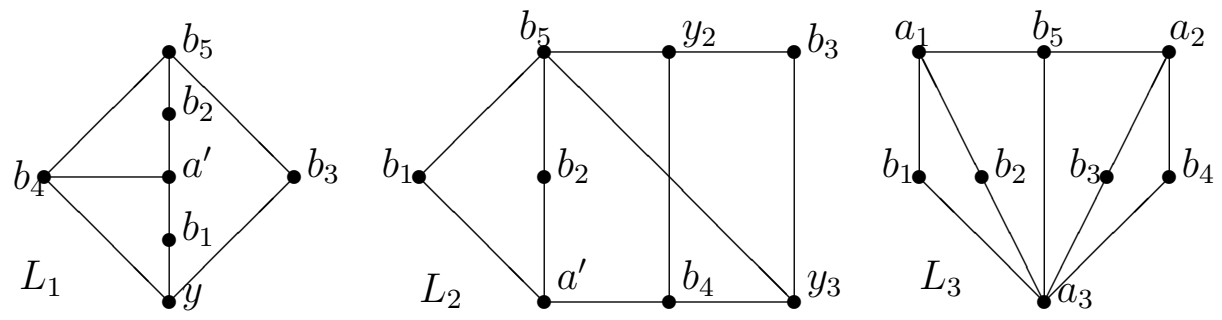

Figure 2.6 The graphs $L_{1}, L_{2}$ and $L_{3}$

Theorem 2.0.13 (Lai, [20]) Let $G$ be a 2-edge-connected graph and let $S \subseteq V(G)$ with $|S| \leq 5$. If $G-S$ is edgeless, and if $G$ does not have an $S$-eulerian subgraph, then $G$ is contractible to a member $L \in \mathcal{F}$ such that $S$ intersects the preimage of every vertex in $B(L)$.

Lemma 2.0.14 Suppose that $G$ is a simple graph which does not contain $K_{4}-e$ as a subgraph. Then the following statements hold.

(i) If $|V(G)|=3$, then $|E(G)| \leq 3$.

(ii) If $|V(G)|=4$, then $|E(G)| \leq 4$.

(iii) If $|V(G)|=5$, then $|E(G)| \leq 6$.

(iv) If $|V(G)|=6$, then $|E(G)| \leq 9$.

(v) If $|V(G)|=7$, then $|E(G)| \leq 12$.

Proof. If $|V(G)|=3$, then $|E(G)| \leq 3$. If $|V(G)|=4$, then $|E(G)| \leq 4$ since $G$ does not contain $K_{4}-e$ as its subgraph. Note that the proofs for (iii),(iv) and (v) are similar. So we only present the proof for $(\mathrm{v})$.

Let $V(G)=\left\{u_{1}, u_{2}, \cdots, u_{7}\right\}$. Without loss of generality, we assume that $d_{G}\left(u_{7}\right)=$ $\Delta(G)$. If $d_{G}\left(u_{7}\right) \leq 3$, then it is clear that (v) holds. Suppose that $d_{G}\left(u_{7}\right)=6$. Then the degree of $u_{i}(i=1,2, \cdots, 6)$ is at most 1 in $G-u_{7}$ since $G$ does not contain $K_{4}-e$ as its subgraph. Thus $\left|E\left(G-u_{7}\right)\right| \leq 3$ and $|E(G)| \leq 6+3=9$.

Suppose that $d_{G}\left(u_{7}\right)=5$ and $u_{7} u_{6} \notin E(G)$. Then the degree of $u_{i}(i=1,2, \cdots, 5)$ is at most 1 in $G-\left\{u_{7}, u_{6}\right\}$. Let $p_{1}=\left|E\left(G-\left\{u_{6}, u_{7}\right\}\right)\right|$. Then $d_{G}\left(u_{6}\right) \leq 5-p_{1}$ since $G$ does not contain $K_{4}-e$ as its subgraph. Thus $|E(G)|=d_{G}\left(u_{6}\right)+d_{G}\left(u_{7}\right)+\left|E\left(G-\left\{u_{6}, u_{7}\right\}\right)\right| \leq$ $5+\left(5-p_{1}\right)+p_{1}=10$. 
Suppose that $d_{G}\left(u_{7}\right)=4$ and $u_{7} u_{6}, u_{7} u_{5} \notin E(G)$. Then the degree of $u_{i}(i=1,2,3,4)$ is at most 1 in $G-\left\{u_{7}, u_{6}, u_{5}\right\}$. Let $p_{2}=\left|E\left(G-\left\{u_{5}, u_{6}, u_{7}\right\}\right)\right|$. Then $\left|\left[\left\{u_{1}, u_{2}, u_{3}, u_{4}\right\},\left\{u_{i}\right\}\right]_{G}\right|$ $\leq 4-p_{2}(i=5,6)$ and $p_{2} \leq 2$ since $G$ does not contain $K_{4}-e$ as its subgraph. If $p_{2}=0$ and $u_{5} u_{6} \in E(G)$, then $\left|\left[\left\{u_{1}, u_{2}, u_{3}, u_{4}\right\},\left\{u_{5}, u_{6}\right\}\right]_{G}\right| \leq 5$. Thus $|E(G)|=$ $d_{G}\left(u_{7}\right)+\left|\left[\left\{u_{1}, u_{2}, u_{3}, u_{4}\right\},\left\{u_{5}, u_{6}\right\}\right]_{G}\right|+\left|E\left(G-\left\{u_{5}, u_{6}, u_{7}\right\}\right)\right|+\left|E\left(G\left[\left\{u_{5}, u_{6}\right\}\right]\right)\right| \leq 4+$ $5+0+1=10$. Otherwise, we have $|E(G)|=d_{G}\left(u_{7}\right)+\left|\left[\left\{u_{1}, u_{2}, u_{3}, u_{4}\right\},\left\{u_{5}\right\}\right]_{G}\right|+$ $\left|\left[\left\{u_{1}, u_{2}, u_{3}, u_{4}\right\},\left\{u_{6}\right\}\right]_{G}\right|+\left|E\left(G-\left\{u_{5}, u_{6}, u_{7}\right\}\right)\right|+\left|E\left(G\left[\left\{u_{5}, u_{6}\right\}\right]\right)\right| \leq 4+\left(4-p_{2}\right)+(4-$ $\left.p_{2}\right)+p_{2}+\left|E\left(G\left[\left\{u_{5}, u_{6}\right\}\right]\right)\right|=12-p_{2}+\left|E\left(G\left[\left\{u_{5}, u_{6}\right\}\right]\right)\right| \leq 12$.

Lemma 2.0.15 Suppose that $G$ is a 2-edge-connected graph with at most 10 vertices, and that $G$ does not contain $K_{4}-e$ as a subgraph. If $|E(G)| \geq 17$, then $G$ is collapsible.

Proof. By Theorem 2.0.5(ii), if $H$ is a simple collapsible subgraph of $G$ with $|V(H)|=4$, then $H$ must contain contain $K_{4}-e$ as a subgraph. Thus we have

if $H$ is a simple collapsible subgraph of $G$, then $|V(H)| \geq 3$ and $|V(H)| \neq 4$.

Let $G^{\prime}$ be the reduction of $G$. Note that $G$ is collapsible if and only if $G^{\prime}=K_{1}$. Suppose, by contradiction, that $G^{\prime} \neq K_{1}$. Then $\kappa^{\prime}\left(G^{\prime}\right) \geq 2$ and $\left|V\left(G^{\prime}\right)\right| \leq 10$. If $\kappa^{\prime}\left(G^{\prime}\right) \geq$ 3 , then by Theorem 2.0.10 and by $|E(G)| \geq 17$, we must have $G^{\prime}=K_{1}$, a contradiction. So we may assume that $\kappa^{\prime}\left(G^{\prime}\right)=2$ and let $X \subseteq E\left(G^{\prime}\right)$ be an edge cut of $G^{\prime}$ with $|X|=2$. Pick an $e \in X$, and denote $[e]=\left\{e^{\prime} \in E\left(G^{\prime}\right) \mid\left\{e, e^{\prime}\right\}\right.$ is an edge cut of $\left.G^{\prime}\right\} \cup\{e\}$. Then for any $\left\{e_{1}, e_{2}\right\} \subseteq[e],\left\{e_{1}, e_{2}\right\}$ is also an edge cut of $G^{\prime}$. Let $|[e]|=k \geq 2$. Then $G^{\prime}$ has $k$ connected subgraphs $G_{1}, G_{2}, \cdots, G_{k}$ such that $G_{i}, G_{i+1}(i=1,2, \cdots, k-1)$ and $G_{1}, G_{k}$ are joined by exactly one edge in $[e]$ (see Figure 2.7 ), and each $G_{i}(i=1,2, \cdots, k)$ is either a $K_{1}$ or a nontrivial 2-edge-connected graph. Thus, if $G_{i}$ is nontrivial, then $\left|V\left(G_{i}\right)\right| \geq 4$, and $F\left(G_{i}\right) \geq 2$ by Theorem 2.0.5(ii). If follows by Theorem 2.0.7(iv) that $\left|E\left(G_{i}\right)\right| \leq 2\left|V\left(G_{i}\right)\right|-4$. Let

$$
t=\mid\left\{G_{i} \mid G_{i} \text { is nontrivial }, 1 \leq i \leq k\right\} \mid .
$$

Since $|V(G)| \leq 10$, we have $0 \leq t \leq 2$. 


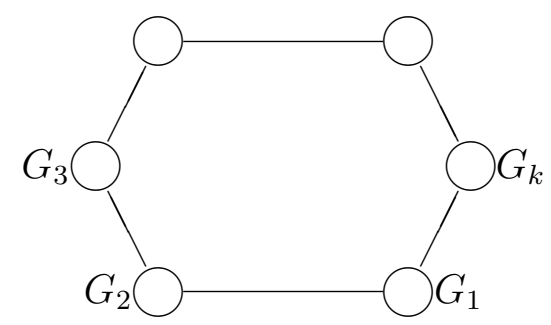

Figure 2.7

Case 1. $t=0$.

Let $V\left(G_{i}\right)=\left\{v_{i}\right\}(i=1,2, \cdots, k)$ and let $s$ be the number of nontrivial vertices in $\left\{v_{1}\right.$, $\left.v_{2}, \cdots, v_{k}\right\}$. Assume that for each $i=1, \cdots, s, v_{i}$ is a nontrivial vertex and $\left|V\left(P I\left(v_{1}\right)\right)\right| \leq$ $\left|V\left(P I\left(v_{2}\right)\right)\right| \leq \cdots\left|V\left(P I\left(v_{s}\right)\right)\right|$. Since $G^{\prime}$ is the reduced graph, we have $k \geq 4$. Since $|V(G)| \leq 10, s \leq 3$. By $(2.1), \mid V\left(P I\left(v_{i}\right) \mid \neq 4\right.$. If $s=3$, then $\left|V\left(P I\left(v_{i}\right)\right)\right|=3$ and $k=4$. Thus $17 \leq|E(G)|=\sum_{i=1}^{3}\left|E\left(P I\left(v_{i}\right)\right)\right|+k=9+4=13$ by Lemma 2.0.14(i), a contradiction. If $s=2$, then $\left|V\left(P I\left(v_{1}\right)\right)\right|=3$, and either $\left|V\left(P I\left(v_{2}\right)\right)\right|=3$ or $\left|V\left(P I\left(v_{2}\right)\right)\right|=5$. Thus $17 \leq|E(G)|=\sum_{i=1}^{2}\left|E\left(P I\left(v_{i}\right)\right)\right|+k=\left\{\begin{array}{ll}3+3+k \leq 6+6=12, & \text { if }\left|V\left(P I\left(v_{2}\right)\right)\right|=3 \\ 3+6+k \leq 9+4=13, & \text { if }\left|V\left(P I\left(v_{2}\right)\right)\right|=5\end{array}\right.$, a contradiction. If $s=1$, then $\left|V\left(P I\left(v_{1}\right)\right)\right|=3,5,6,7$. Thus $|E(G)|=\left|E\left(P I\left(v_{1}\right)\right)\right|+$ $k=\left\{\begin{array}{ll}3+k \leq 3+8=11, & \text { if }\left|V\left(P I\left(v_{1}\right)\right)\right|=3 \\ 6+k \leq 6+6=12, & \text { if }\left|V\left(P I\left(v_{1}\right)\right)\right|=5 \\ 9+k \leq 9+5=14, & \text { if }\left|V\left(P I\left(v_{1}\right)\right)\right|=6 \\ 12+k \leq 12+4=16, & \text { if }\left|V\left(P I\left(v_{1}\right)\right)\right|=7\end{array}\right.$, contrary to the assumption that $|E(G)| \geq 17$.

Case 2. $\quad t=1$.

Without loss of generality, we assume that $G_{1}$ is the nontrivial subgraph in $G^{\prime}$. Then $\left|E\left(G_{1}\right)\right| \leq 2\left|V\left(G_{1}\right)\right|-4$. Since $\left|V\left(G_{1}\right)\right| \geq 4$ and $|V(G)| \leq 10$, we have $2 \leq k \leq 7$.

Case 2.1. $8 \leq\left|V\left(G_{1}\right)\right| \leq 9$

In this case, $G_{1} \subseteq G, k \leq 11-\left|V\left(G_{1}\right)\right|$ and for each $v \in V\left(G^{\prime}\right)-V\left(G_{1}\right)$, $v$ must be trivial. Thus $17 \leq|E(G)|=\left|E\left(G_{1}\right)\right|+k \leq\left(2\left|V\left(G_{1}\right)\right|-4\right)+\left(11-\left|V\left(G_{1}\right)\right|\right)=7+\left|V\left(G_{1}\right)\right| \leq$ 
16 , a contradiction.

Case 2.2. $6 \leq\left|V\left(G_{1}\right)\right| \leq 7$

If $v$ is trivial for each $v \in V\left(G^{\prime}\right)$, then $k \leq 11-\left|V\left(G_{1}\right)\right|$ and $17 \leq|E(G)|=\left|E\left(G_{1}\right)\right|+$ $k \leq 7+\left|V\left(G_{1}\right)\right| \leq 14$, a contradiction. So there exists some vertex $v \in V\left(G^{\prime}\right)$ such that $v$ is not trivial. By $(2.1),|V(P I(v))| \neq 4$ and $|V(P I(v))| \geq 3$. Note that $|V(G)|=$ $\left|V\left(G_{1}\right)\right|+|V(P I(v))|+(k-2) \leq 10$ and $k \geq 2$. Then we have $|V(P I(v))|=3$. By Lemma 2.0.14(i), $|E(P I(v))|=3$. Thus $17 \leq|E(G)|=\left|E\left(G_{1}\right)\right|+|E(P I(v))|+k \leq$ $\left(2\left|V\left(G_{1}\right)\right|-4\right)+3+\left(12-\left|V\left(G_{1}\right)\right|-|V(P I(v))|\right)=8+\left|V\left(G_{1}\right)\right| \leq 15$, a contradiction.

Case 2.3. $\left|V\left(G_{1}\right)\right|=5$

If $v$ is trivial for each $v \in V\left(G^{\prime}\right)$, then $k \leq 6$ and $|E(G)|=\left|E\left(G_{1}\right)\right|+k \leq\left(2\left|V\left(G_{1}\right)\right|-\right.$ $4)+k \leq 6+6=12$, a contradiction. So $V\left(G^{\prime}\right)$ must have a nontrivial vertex. Let $q_{1}=\mid\left\{v \in V\left(G^{\prime}\right) \mid v\right.$ is a nontrivial vertex $\} \mid$.

Suppose that $q_{1}=1$ and $v$ is a nontrivial vertex. Then $|V(P I(v))|=3$ or 5 by (2.1). If $|V(P I(v))|=3$, then $k \leq 4$. Thus $17 \leq|E(G)|=\left|E\left(G_{1}\right)\right|+|E(P I(v))|+k \leq$ $\left(2\left|V\left(G_{1}\right)\right|-4\right)+3+4=6+7=13$, a contradiction. If $|V(P I(v))|=5$, then $k=2$. Thus $17 \leq|E(G)|=\left|E\left(G_{1}\right)\right|+|E(P I(v))|+k \leq\left(2\left|V\left(G_{1}\right)\right|-4\right)+6+2=6+8=14$, a contradiction.

Suppose that $q_{1} \geq 2$ and that $v_{2}, \cdots, v_{q_{1}+1}$ are nontrivial vertices. By $(2.1)$, we have $k=2, q_{1}=2,\left|V\left(P I\left(v_{i}\right)\right)\right|=3(i=2,3)$. Thus $17 \leq|E(G)|=\left|E\left(G_{1}\right)\right|+\left|E\left(P I\left(v_{2}\right)\right)\right|+$ $\left|E\left(P I\left(v_{3}\right)\right)\right|+k \leq\left(2\left|V\left(G_{1}\right)\right|-4\right)+3+3+2=6+8=14$, a contradiction.

Case 2.4. $\left|V\left(G_{1}\right)\right|=4$

If $v$ is trivial for each $v \in V\left(G^{\prime}\right)$, then $k \leq 7$ and $17 \leq|E(G)|=\left|E\left(G_{1}\right)\right|+k \leq$ $\left(2\left|V\left(G_{1}\right)\right|-4\right)+k=4+7=11$, a contradiction. So $V\left(G^{\prime}\right)$ must have a nontrivial vertex. Let $q_{2}=\mid\left\{v \in V\left(G^{\prime}\right) \mid v\right.$ is a nontrivial vertex $\} \mid$.

Suppose that $q_{2}=1$ and $v$ is a nontrivial vertex. Then $|V(P I(v))|=3,5,6$ by (2.1). If $|V(P I(v))|=3$, then $k \leq 5$. Thus $17 \leq|E(G)|=\left|E\left(G_{1}\right)\right|+|E(P I(v))|+k \leq$ $\left(2\left|V\left(G_{1}\right)\right|-4\right)+3+5=4+8=12$, a contradiction. If $|V(P I(v))|=5$, then $k \leq 3$. 
Thus $17 \leq|E(G)|=\left|E\left(G_{1}\right)\right|+|E(P I(v))|+k \leq\left(2\left|V\left(G_{1}\right)\right|-4\right)+6+3=4+9=13$, a contradiction. If $|V(P I(v))|=6$, then $k=2$. Thus $17 \leq|E(G)|=\left|E\left(G_{1}\right)\right|+|E(P I(v))|+$ $k \leq\left(2\left|V\left(G_{1}\right)\right|-4\right)+9+2=4+11=15$, a contradiction.

Suppose that $q_{2} \geq 2$ and that $v_{2}, \cdots, v_{q_{2}+1}$ are nontrivial vertices. By $(2.1)$, we have $k \leq 3, q_{2}=2$ and $\left|V\left(P I\left(v_{i}\right)\right)\right|=3(i=2,3)$. Thus $17 \leq|E(G)|=\left|E\left(G_{1}\right)\right|+\left|E\left(P I\left(v_{1}\right)\right)\right|+$ $\left|E\left(P I\left(v_{2}\right)\right)\right|+k \leq\left(2\left|V\left(G_{1}\right)\right|-4\right)+3+3+3=4+9=13$, a contradiction.

Case 3. $\quad t=2$.

Without loss of generality, we assume that $G_{1}, G_{2}$ are the nontrivial subgraphs in $G^{\prime}$. If there exists $v \in V\left(G_{1}\right) \cup V\left(G_{2}\right)$ such that $v$ is a nontrivial vertex in $G^{\prime}$, then $k=2, V\left(G^{\prime}\right)=V\left(G_{1}\right) \cup V\left(G_{2}\right),\left|V\left(G_{1}\right)\right|=\left|V\left(G_{2}\right)\right|=4$ and $|P I(v)|=3$. Thus we have $17 \leq|E(G)|=\left|E\left(G_{1}\right)\right|+\left|E\left(G_{2}\right)\right|+|E(P I(v))|+k \leq 4+4+3+2=13$, a contradiction. So $V\left(G_{i}\right) \subseteq V(G)(i=1,2)$. Note that $\left|V\left(G_{i}\right)\right| \geq 4$, we have $k \leq 4$. Since $\left|V\left(G_{1}\right)\right|+\left|V\left(G_{2}\right)\right| \leq 10$ and $\left|E\left(G_{i}\right)\right| \leq 2\left|V\left(G_{i}\right)\right|-4$, we have $\left|E\left(G_{1}\right)\right|+\left|E\left(G_{2}\right)\right| \leq 12$. Thus $17 \leq|E(G)|=\left|E\left(G_{1}\right)\right|+\left|E\left(G_{2}\right)\right|+k \leq 12+4=16$, a contradiction. 


\section{Chapter 3}

\section{Eulerian subgraphs and Hamilton-connected line graphs}

\subsection{Introduction}

For integers $k \geq 0$ and $l>0$, let $C(l, k)$ denote the class of 2-edge-connected graphs of order $n$ such that $G \in C(l, k)$ if and only if for every edge cut $S \subseteq E(G)$ with $|S| \leq 3$, each component of $G-S$ has order at least $\frac{n-k}{l}$.

Catlin and Li, and Broersma and Xiong proved the following results concerning when a graph in the family $C(l, k)$ is supereulerian.

Theorem 3.1.1 (Catlin and Li, [9]) If $G \in C(5,0)$, then $G \in \mathcal{S L}$ if and only if $G$ is not contractible to $K_{2,3}$.

Theorem 3.1.2 (Broersma and Xiong, [2]) If $G \in C(5,2)$ and $n \geq 13$, then $G \in \mathcal{S L}$ if and only if $G$ is not contractible to $K_{2,3}$ or $K_{2,5}$.

In this chapter, we further study the distribution of the small degree vertices in the 
reduction of a graph, and sharpen Theorems 3.1.1 and 3.1.2, as shown in Theorem 3.1.3 and Corollary 3.1.4.

Theorem 3.1.3 If $G \in C(6,0)$, then $G \in \mathcal{S L}$ if and only if $G$ is not contractible to $K_{2,3}, K_{2,5}$ or $K_{2,3}(e)$, where $e \in E\left(K_{2,3}\right)$.

Note that when $n \geq 6 k+1, C(5, k) \subseteq C(6,0)$. So we have

Corollary 3.1.4 If $G \in C(5, k)$ and $n \geq 6 k+1$, then $G \in \mathcal{S L}$ if and only if $G$ is not contractible to $K_{2,3}$ or $K_{2,5}$.

We investigate the Hamilton-connectedness of line graphs of graphs in $C(l, k)$ and get the following.

Theorem 3.1.5 If $G \in C(6,0)$ and $n \geq 7$, then $L(G)$ is Hamilton-connected if and only if $\kappa(L(G)) \geq 3$.

Corollary 3.1.6 If $G \in C(5, k)$ and $n \geq \max \{6 k+1,6\}$, then $L(G)$ is Hamiltonconnected if and only if $\kappa(L(G)) \geq 3$.

\subsection{Proofs of Theorems 3.1.3 and 3.1.5}

Proof of Theorem 3.1.3. Let $G^{\prime}$ be the reduction of $G$. If $G^{\prime}=K_{1}$, then $G \in \mathcal{S L}$ by Theorem 2.0.4(iv). Next we suppose that $G^{\prime} \neq K_{1}$. Then $G^{\prime}$ is 2-edge-connected and nontrivial. Denote $d_{i}=\left|D_{i}\left(G^{\prime}\right)\right|(i \geq 2)$.

If $d_{2}+d_{3} \geq 7$, then we assume that $v_{1}, v_{2}, \cdots, v_{7}$ are the vertices of $V\left(G^{\prime}\right)$ in $D_{2}\left(G^{\prime}\right) \cup$ $D_{3}\left(G^{\prime}\right)$, i.e. $d_{G^{\prime}}\left(v_{i}\right) \leq 3$ for each $i$, and the corresponding pre-images are $H_{1}, H_{2}, \cdots, H_{7}$. 
Each $H_{i}$ is joined to the rest of $G$ by an edge cut consisting of $d_{G^{\prime}}\left(v_{i}\right) \leq 3$ edges. By the hypothesis of Theorem 3.1.3, $\left|V\left(H_{i}\right)\right| \geq \frac{n}{6}$, and

$$
n=|V(G)| \geq \sum_{i=1}^{7}\left|V\left(H_{i}\right)\right| \geq \frac{7 n}{6},
$$

a contraction. Therefore we assume $d_{2}+d_{3} \leq 6$, and when $d_{2}+d_{3}=6, V\left(G^{\prime}\right)=$ $D_{2}\left(G^{\prime}\right) \cup D_{3}\left(G^{\prime}\right)$. We break the proof into two cases.

Case 1. $F\left(G^{\prime}\right) \leq 2$.

By $\kappa^{\prime}\left(G^{\prime}\right) \geq 2$ and by Theorem 2.0.6, $G^{\prime} \in \mathcal{S} \mathcal{L}$ or $G^{\prime}=K_{2, s}$ where $s \geq 3$ is an odd integer. In the former case, $G \in \mathcal{S} \mathcal{L}$ by Theorem 2.0.4(iv). In the latter, $s=3$ or $s=5$ by $d_{2}+d_{3} \leq 6$.

Case 2. $F\left(G^{\prime}\right) \geq 3$.

Note that $\left|V\left(G^{\prime}\right)\right|=\sum_{i \geq 2} d_{i}, 2\left|E\left(G^{\prime}\right)\right|=\sum_{i \geq 2} i d_{i}$. By Theorem 2.0.7(iv), we have the following

$$
2 d_{2}+d_{3} \geq 10+\sum_{i \geq 5}(i-4) d_{i}
$$

Since $d_{2}+d_{3} \leq 6, d_{2} \geq 4$. We distinguish two cases to complete the proof.

Case 2.1. $d_{2}=4$.

By $(3.1)$ and $d_{2}+d_{3} \leq 6, d_{3}=2$. Thus $V\left(G^{\prime}\right)=D_{2}\left(G^{\prime}\right) \cup D_{3}\left(G^{\prime}\right)$. Let $D_{2}\left(G^{\prime}\right)=$ $\left\{u_{1}, u_{2}, u_{3}, u_{4}\right\}$ and $D_{3}\left(G^{\prime}\right)=\left\{v_{1}, v_{2}\right\}$. If $v_{1} v_{2} \in E\left(G^{\prime}\right)$, then $E\left(G^{\prime}\right)=\left\{u_{1} u_{2}, u_{2} v_{2}, v_{2} u_{3}\right.$, $\left.u_{3} u_{4}, u_{4} v_{1}, v_{1} u_{1}, v_{1} v_{2}\right\}$ by Theorem 2.0.4(ii). Thus $G^{\prime} \in \mathcal{S} \mathcal{L}$ and so $G \in \mathcal{S} \mathcal{L}$. If $v_{1} v_{2} \notin$ $E\left(G^{\prime}\right)$, then $G^{\prime}=K_{2,3}(e)$, where $e \in E\left(K_{2,3}\right)$.

Case 2.2. $d_{2}=5$ or 6 .

If $d_{2}=5$, then $d_{3}=0$ and $d_{i}=0(i \geq 5)$ by (3.1). If $d_{2}=6$, then $d_{i}=0(i \geq 3)$. Thus $G^{\prime} \in \mathcal{S} \mathcal{L}$ and so $G \in \mathcal{S} \mathcal{L}$.

Proof of Theorem 3.1.5. It is trivial that $\kappa(L(G)) \geq 3$ if $L(G)$ is Hamilton-connected. 
So we only need to prove that $L(G)$ is Hamilton-connected when $\kappa(L(G)) \geq 3$.

Let $e_{1}, e_{2} \in E(G)$. By Theorem 1.0.2, Proposition 1.0.3 and Lemma 2.0.12, we need to prove $G\left(e_{1}, e_{2}\right) \in \mathcal{C} \mathcal{L}$. Let $G^{\prime}$ be the reduction of $G\left(e_{1}, e_{2}\right)$. By Theorem 2.0.4(iv), it suffices to prove that $G^{\prime}=K_{1}$. Suppose that $G^{\prime} \neq K_{1}$. Then $G^{\prime}$ is 2-edge-connected and nontrivial. Denote $d_{i}=\left|D_{i}\left(G^{\prime}\right)\right|(i \geq 2)$.

If $d_{2} \geq 3$, then there exists $v \in D_{2}\left(G^{\prime}\right)-\left\{v\left(e_{1}\right), v\left(e_{2}\right)\right\}$ such that $d_{G^{\prime}}(v)=2$. Let $H$ be the pre-image of $v$ in $G\left(e_{1}, e_{2}\right)$. Then $H$ is joined to the rest of $G\left(e_{1}, e_{2}\right)$, therefore of $G$, by an edge-cut consisting of $d_{G^{\prime}}(v)=2$ edges. By the hypothesis of Theorem 3.1.5, $|V(H)| \geq \frac{n}{6}>1$. Thus $\kappa(L(G)) \leq 2$, a contradiction. So $d_{2} \leq 2$.

If $d_{2}+d_{3} \geq 9$, then $\left|D_{2}\left(G^{\prime}\right) \cup D_{3}\left(G^{\prime}\right)-\left\{v\left(e_{1}\right), v\left(e_{2}\right)\right\}\right| \geq 7$. We assume that $v_{1}, v_{2}, \cdots, v_{7}$ are the vertices of $V\left(G^{\prime}\right)$ in $D_{2}\left(G^{\prime}\right) \cup D_{3}\left(G^{\prime}\right)-\left\{v\left(e_{1}\right), v\left(e_{2}\right)\right\}$, i.e. $d_{G^{\prime}}\left(v_{i}\right) \leq 3$ for each $i$, and the corresponding pre-images are $H_{1}, H_{2}, \cdots, H_{7}$. Each $H_{i}$ is joined to the rest of $G\left(e_{1}, e_{2}\right)$, therefore to the rest of $G$, by an edge cut consisting of $d_{G^{\prime}}\left(v_{i}\right) \leq 3$ edges. By the hypothesis of Theorem 3.1.5, $\left|V\left(H_{i}\right)\right| \geq \frac{n}{6}$, and

$$
n=|V(G)| \geq \sum_{i=1}^{7}\left|V\left(H_{i}\right)\right| \geq \frac{7 n}{6},
$$

a contraction. So $d_{2}+d_{3} \leq 8$, and when $d_{2}+d_{3}=8, V\left(G^{\prime}\right)=D_{2}\left(G^{\prime}\right) \cup D_{3}\left(G^{\prime}\right)$.

Suppose that $F\left(G^{\prime}\right) \geq 3$, i.e., $2\left|V\left(G^{\prime}\right)\right|-\left|E\left(G^{\prime}\right)\right| \geq 5$ by Theorem 2.0.7(iv). Note that $\left|V\left(G^{\prime}\right)\right|=\sum_{i \geq 2} d_{i}, 2\left|E\left(G^{\prime}\right)\right|=\sum_{i \geq 2} i d_{i}$, we have the following

$$
2 d_{2}+d_{3} \geq 10+\sum_{i \geq 5}(i-4) d_{i}
$$

By (3.2) and $d_{2}+d_{3} \leq 8, d_{2}=2$ and $d_{3}=6$. Thus $\left|V\left(G^{\prime}\right)\right|=8$. As $G^{\prime}$ is 2-edgeconnected nontrivial reduced graph, we have $G^{\prime}=K_{2,3}$ by Lemma 2.0.11, a contradiction. So $F\left(G^{\prime}\right) \leq 2$.

As $G^{\prime}$ is 2-edge-connected and $d_{2} \leq 2, G \neq K_{2}, K_{2, t}(t \geq 1)$. Thus by Theorem 2.0.5(iii), $G^{\prime}=K_{1}$, a contradiction. 


\subsection{Applications}

Theorem 3.1.3 and Corollary 3.1.6 have a number of applications.

Theorem 3.3.1 (Zhan, [27]) Let $G$ be a graph. If $\kappa^{\prime}(G) \geq 4$, then $L(G)$ is Hamiltonconnected.

Theorem 3.3.2 (Broersma and Xiong, [2]) Let $G$ be a 2-edge-connected simple graph with $n \geq 13$ vertices. If $\delta(G) \geq 4$ and if

$$
\min \{\max \{d(x), d(y)\} \mid x y \in E(G)\} \geq \frac{n-2}{5}-1,
$$

then $G \in \mathcal{S L}$ if and only if $G$ is not contractible to $K_{2,3}$ or $K_{2,5}$.

Theorem 3.3.3 Let $G$ be a 2-edge-connected simple graph. If $\delta(G) \geq 4$ and if

$$
\min \{\max \{d(x), d(y)\} \mid x y \in E(G)\} \geq \frac{n}{6}-1,
$$

then $G \in \mathcal{S L}$ if and only if $G$ is not contractible to $K_{2,3}, K_{2,5}$ or $K_{2,3}(e)$.

Proof. Let $S$ be an edge cut of $G$ with $|S| \leq 3$, and let $G_{1}$ and $G_{2}$ be the two components of $G-S$ with $\left|V\left(G_{1}\right)\right| \leq\left|V\left(G_{2}\right)\right|$. It is sufficient to prove that $\left|V\left(G_{1}\right)\right| \geq \frac{n}{6}$ by Theorem 3.1.3. Since $\delta(G) \geq 4, G_{1}$ has at least an edge, say $u v$, such that both of $u, v$ are not incident with any edges of $S$. By the hypothesis of Theorem 3.3.3,

$$
\left|V\left(G_{1}\right)\right| \geq \max \{d(u), d(v)\}+1 \geq \frac{n}{6}
$$

Thus Theorem 3.3.3 follows from Theorem 3.1.3.

Obviously, Theorem 3.3.3 improves Theorem 3.3.2 and the following results.

Corollary 3.3.4 Let $G$ be a 2-edge-connected simple graph with $n \geq 6 k+1$ vertices. If $\delta(G) \geq 4$ and if

$$
\min \{\max \{d(x), d(y)\} \mid x y \in E(G)\} \geq \frac{n-k}{5}-1,
$$

then $G \in \mathcal{S L}$ if and only if $G$ is not contractible to $K_{2,3}$ or $K_{2,5}$. 
Corollary 3.3.5 $G$ be a 2-edge-connected simple graph. If $\delta(G) \geq 4$ and if every edge $u v \in E(G)$ satisfies

$$
d(u)+d(v) \geq \frac{n}{3}-2,
$$

then $G \in \mathcal{S L}$ if and only if $G$ is not contractible to $K_{2,3}, K_{2,5}$ or $K_{2,3}(e)$.

Corollary 3.3.6 Let $G$ be a 2-edge-connected simple graph of order $n>24$. If

$$
\delta(G) \geq \frac{n}{6}-1,
$$

then $G \in \mathcal{S L}$ if and only if $G$ is not contractible to $K_{2,3}, K_{2,5}$ or $K_{2,3}(e)$. 


\section{Chapter 4}

\section{Supereulerian planar graphs}

\subsection{Introduction}

In 1989, Catlin [5] conjectured that if $F(G) \leq 3$ and if $G$ is 3-edge-connected, then $G$ is collapsible if and only if the reduction of $G$ is not the Petersen graph. Noting that planar graphs cannot be contracted to the Petersen graph, we in this chapter prove the following.

Theorem 4.1.1 Let $G$ be a 3-edge-connected planar graph. If $F(G) \leq 3$, then $G$ is collapsible.

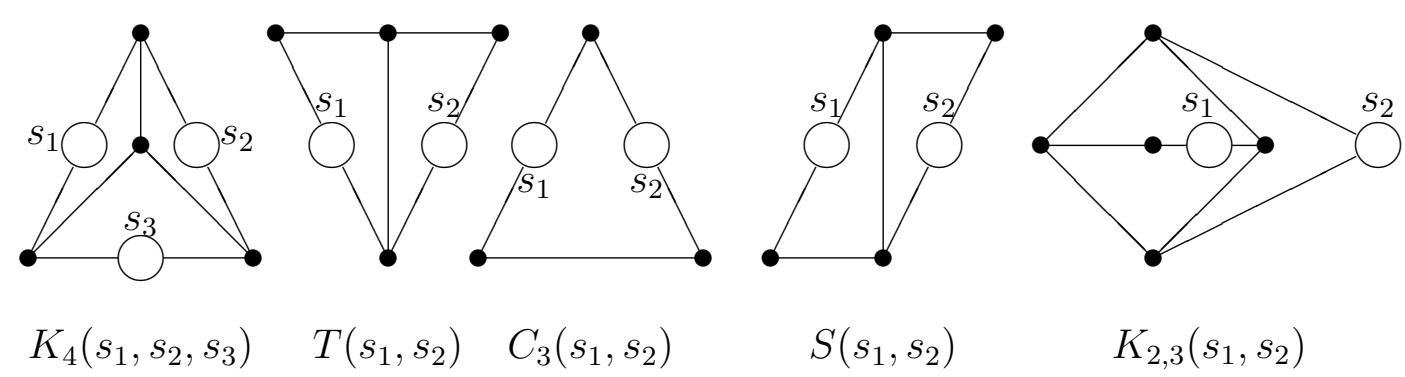

Figure 4.1

Let $s_{i} \geq 1(i=1,2,3)$ be integers. Denote $K_{4}\left(s_{1}, s_{2}, s_{3}\right), T\left(s_{1}, s_{2}\right), C_{3}\left(s_{1}, s_{2}\right), S\left(s_{1}, s_{2}\right)$ and $K_{2,3}\left(s_{1}, s_{2}\right)$ to be the graphs depicted in Figure 4.1, where the $s_{i}(i=1,2,3)$ vertices 
and the two vertices connected by the two lines shown in each of the graphs forms a $K_{2, s_{i}}$ graph. Denote

$\mathcal{F}_{1}=\left\{K_{4}\left(s_{1}, s_{2}, s_{3}\right), T\left(s_{1}, s_{2}\right), C_{3}\left(s_{1}, s_{2}\right), S\left(s_{1}, s_{2}\right), K_{2,3}\left(s_{1}, s_{2}\right) \mid s_{i} \geq 1(i=1,2,3)\right.$ is an integer $\}$

and $\mathcal{F}=\mathcal{F}_{1} \cup\left\{K_{2, t} \mid t \geq 2\right\}$. Clearly, each graph $G$ in $\mathcal{F}$ is reduced and $F(G) \leq 3$.

Theorem 4.1.2 Let $G$ be a 2-edge-connected planar graph. If $F(G) \leq 3$, then either $G$ is collapsible or the reduction of $G$ is a graph in $\mathcal{F}$.

Theorem 4.1.3 Let $G$ be an essentially 3-edge-connected planar reduced graph with $\kappa^{\prime}(G) \geq$ 2. If $F(G) \leq 5$, then $G$ has a dominating Eulerian subgraph $H$ such that $D_{3}^{*}(G) \subseteq H$.

Applying Theorem 4.1.3, we can improve Theorems 3.1.1 and 3.1.2 within planar graphs.

Theorem 4.1.4 Let $G$ be a simple planar graph of order $n$ with $\kappa^{\prime}(G) \geq 3$. If $G \in$ $C(16,0)$, then $G$ is supereulerian.

In [3], Cai considered this problem: Find the best possible bound $f(n)$ for a simple graph $G$ with $n$ vertices such that if $|E(G)| \geq f(n)$, then $G$ is supereulerian. Let $Q_{3}$ denote the cube $\left(K_{2} \times C_{4}\right)$ and $Q_{3}-v$ denote the cube minus a vertex. Cai proved the following result.

Theorem 4.1.5 (Cai, [3]) Let $G$ be a simple graph of order $n \geq 5$ and $\kappa^{\prime}(G) \geq 2$. If

$$
|E(G)| \geq\left(\begin{array}{c}
n-4 \\
2
\end{array}\right)+6,
$$

then exactly one of the following holds:

(i) $G$ is supereulerian.

(ii) $G$ can be contracted to a $K_{2,3}$.

(iii) $G$ is the graph $K_{2,5}$ or $Q_{3}-v$. 
In [3], Cai conjectured that when restricted to the 3-edge-connected simple graphs, the lower bound can be improved. In [7] and [11], Catlin and Chen settled this conjecture.

Theorem 4.1.6 (Catlin and Chen, [7], [11]) Let $G$ be a simple graph with $n \geq 11$ vertices and with $\kappa^{\prime}(G) \geq 3$. If

$$
|E(G)| \geq\left(\begin{array}{c}
n-9 \\
2
\end{array}\right)+16
$$

then $G$ is collapsible.

Graphs contractible to the Petersen graph indicate the sharpness of this result. In this chapter, we prove the following related results among planar graphs.

Theorem 4.1.7 Let $G$ be a planar graph with $n$ vertices, with $\kappa^{\prime}(G) \geq 3$ and $g(G) \geq 4$. If $|E(G)| \geq 2 n-5$, then $G$ is collapsible.

Theorem 4.1 .7 cannot be relaxed to 2-edge-connected planar graphs since $K_{2, t}$ is not collapsible.

Theorem 4.1.8 Let $G$ be a planar graph with $\kappa^{\prime}(G) \geq 3$ such that every edge of $G$ is in a face of degree at most 6 . If either $G$ has at most two faces of degree 5 and no faces of degree bigger than 5, or $G$ has exactly one face of degree 6 and no other faces of degree bigger than 4 , then $G$ is collapsible.

Theorem 4.1.8 is related to a former conjecture of Paulraja ([23], [24]): If $G$ is a 2-connected graph with $\delta(G) \geq 3$ such that every edge of $G$ lies in a cycle of length at most 4 , then $G$ is supereulerian. This conjecture was proved in [19].

Theorem 4.1.9 If $G$ is a 2-edge-connected simple planar graph with order $n \geq 6$ and $|E(G)| \geq 3 n-8$, then $F(G)=0$. 
Theorem 4.1.10 If $G$ is a 2-edge-connected simple planar graph with $n \geq 9$ vertices and $|E(G)| \geq 3 n-12$ edges, then exactly one of the following holds:

(i) $G$ is supereulerian.

(ii) $G$ has a maximal collapsible subgraph $H$ with order $n-4$ such that $G / H$ is a $K_{2,3}$.

In this chapter, we present the proofs of Theorem 4.1.1 and Theorem 4.1.2 in Section 4.2. Theorems 4.1 .2 and 4.1 .1 will be applied to prove Theorems 4.1.3 and 4.1.4 in Section 4.3, to prove Theorems 4.1.7 and 4.1.8 in Section 4.4, respectively. The proofs for Theorems 4.1.9 and 4.1.10 are in Section 4.5.

\subsection{Proofs of Theorem 4.1.1 and Theorem 4.1.2}

Proof of Theorem 4.1.1. Let $G$ be a 3-edge-connected planar graph with $F(G) \leq 3$ and let $G^{\prime}$ denote its reduction. By Theorem 2.0.4(iv), we only need to show that $G^{\prime}=K_{1}$.

By contradiction. Suppose that $G^{\prime}$ is nontrivial. Since $G$ is 3-edge-connected and planar, $G^{\prime}$ is also 3-edge-connected and planar. Since every spanning tree of $G$ will become a connected spanning subgraph in any contraction of $G, F\left(G^{\prime}\right) \leq F(G) \leq 3$. We may assume that $G^{\prime}$ is embedded on the plane.

By Theorem 2.0.4(ii) and (iii), $G^{\prime}$ is reduced and cannot have any cycles of length 2 or 3 , and so $G^{\prime}$ is a simple plane graph each of whose face has degree at least 4 . Let $f$ denote the number of faces of $G^{\prime}$ and let $f_{i}$ denote the number of faces of $G^{\prime}$ having degree $i$, where $i \geq 1$ is an integer. Note that $f_{1}=f_{2}=f_{3}=0$, and so we have

$$
4 f+\sum_{i=5}^{\infty}(i-4) f_{i}=\sum_{i=4}^{\infty} i f_{i}=2\left|E\left(G^{\prime}\right)\right| .
$$

By (4.1) and by Euler's formula,

$$
2\left|E\left(G^{\prime}\right)\right|=2\left|V\left(G^{\prime}\right)\right|+2 f-4=2\left|V\left(G^{\prime}\right)\right|+\left|E\left(G^{\prime}\right)\right|-4-\frac{1}{2} \sum_{i=5}^{\infty}(i-4) f_{i} .
$$


Thus $\left|E\left(G^{\prime}\right)\right|=2\left|V\left(G^{\prime}\right)\right|-4-\frac{1}{2} \sum_{i=5}^{\infty}(i-4) f_{i}$. On the other hand, since $F\left(G^{\prime}\right) \leq 3$ and by Theorem 2.0.7(iv), $\left|E\left(G^{\prime}\right)\right| \geq 2\left|V\left(G^{\prime}\right)\right|-5$. It follows that

$$
2\left|V\left(G^{\prime}\right)\right|-5 \leq\left|E\left(G^{\prime}\right)\right|=2\left|V\left(G^{\prime}\right)\right|-4-\frac{1}{2} \sum_{i=5}^{\infty}(i-4) f_{i} .
$$

If $\left|E\left(G^{\prime}\right)\right|=2\left|V\left(G^{\prime}\right)\right|-4$, then by Theorem 2.0.7(iv), $F\left(G^{\prime}\right)=2$, and so by Theorem 2.0.5(iii) and by the fact that $G^{\prime}$ is 3-edge-connected, $G^{\prime}$ must be a collapsible graph. Thus $G^{\prime}=K_{1}$ by Theorem 2.0.4(iv). This contradicts the assumption that $G^{\prime}$ is nontrivial. Therefore, to obtain our final contradiction, we only need to show that $\left|E\left(G^{\prime}\right)\right|=2\left|V\left(G^{\prime}\right)\right|-5$ is impossible.

If $\left|E\left(G^{\prime}\right)\right|=2\left|V\left(G^{\prime}\right)\right|-5$, then by (4.3), we have

$$
\text { either } f_{4}=f-2 \text { and } f_{5}=2 \text {, or } f_{4}=f-1 \text { and } f_{6}=1 \text {. }
$$

Since $\kappa^{\prime}\left(G^{\prime}\right) \geq 3$, we must have $f_{4} \geq 1$. Let $C=v_{1} v_{2} v_{3} v_{4} v_{1}$ denote a 4-cycle of $G^{\prime}$ and consider $G_{\pi}^{\prime}$. Since $\kappa^{\prime}\left(G^{\prime}\right) \geq 3, G_{\pi}^{\prime}$ is connected. Moreover,

if $\kappa^{\prime}\left(G_{\pi}^{\prime}\right) \leq 2$, then $e_{\pi}=w_{1} w_{2}$ is in an edge cut of size at most 2 in $G_{\pi}^{\prime}$.

Suppose first that $\kappa^{\prime}\left(G_{\pi}^{\prime}\right) \geq 2$. Then by Theorem 2.0.7(ii), $F\left(G_{\pi}^{\prime}\right) \leq 2$. It follows by Theorem 2.0.5(iii) that either $G_{\pi}^{\prime}$ is collapsible, whence $G^{\prime}$ is collapsible by Theorem 2.0.7(i), contrary to the assumption that $G^{\prime}$ is reduced; or the reduction of $G_{\pi}^{\prime}$ is a $K_{2, t}$ for some integer $t \geq 2$, whence $G^{\prime}$ has an edge cut of size 2, contrary to the fact that $\kappa^{\prime}\left(G^{\prime}\right) \geq 3$.

Therefore by (4.5), $e_{\pi}$ must be the only cut edge of $G_{\pi}^{\prime}$. Let $G_{1}^{\prime}$ and $G_{2}^{\prime}$ be the two components of $G_{\pi}^{\prime}-e_{\pi}$ with $w_{1} \in V\left(G_{1}^{\prime}\right)$ and $w_{2} \in V\left(G_{2}^{\prime}\right)$. Then $G^{\prime}-E(C)$ has two components $G_{1}$ and $G_{2}$ with $v_{1}, v_{3} \in V\left(G_{1}\right)$ and $v_{2}, v_{4} \in V\left(G_{2}\right)$ such that $G_{1}^{\prime}$ can be obtained from $G_{1}$ by identifying $v_{1}$ and $v_{3}$, and $G_{2}^{\prime}$ can be obtained from $G_{2}$ by identifying $v_{2}$ and $v_{4}$. Since $G^{\prime}$ is reduced, both $G_{1}$ and $G_{2}$ are reduced. 
If $F\left(G_{1}\right) \leq 2$, then by Theorem 2.0.5(iii) and by the fact that $G_{1}$ is reduced, $G_{1}$ is either a $K_{2}$ or a $K_{2, t}$, for some integer $t \geq 1$. If $G_{1}=K_{2}$, then $G^{\prime}$ has a 3-cycle, contrary to Theorem 2.0.4(ii); if $G_{1}=K_{2, t}$, then $G^{\prime}$ has an edge cut of two edges, contrary to the assumption that $\kappa^{\prime}\left(G^{\prime}\right) \geq 3$. Therefore $F\left(G_{1}\right) \geq 3$. Similarly, $F\left(G_{2}\right) \geq 3$.

By Theorem 2.0.7(iv), $\left|E\left(G_{i}\right)\right| \leq 2\left|V\left(G_{i}\right)\right|-5$ for both $i=1$ and $i=2$. It follows by $F\left(G^{\prime}\right) \leq 3$ and by Theorem 2.0.7(iv) that

$$
\begin{aligned}
2\left|V\left(G^{\prime}\right)\right|-9 & =\left|E\left(G^{\prime}\right)\right|-4=\sum_{i=1}^{2}\left|E\left(G_{i}\right)\right| \\
& \leq 2\left(\sum_{i=1}^{2}\left|V\left(G_{i}\right)\right|-5\right)=2\left|V\left(G^{\prime}\right)\right|-10
\end{aligned}
$$

a contradiction. Thus $G^{\prime}$ must be a $K_{1}$ and so $G$ is collapsible.

Proof of Theorem 4.1.2. Suppose that $G$ is not collapsible, and $G^{\prime}$ is the reduction of $G$. Then $G^{\prime} \neq K_{1}$ and $F\left(G^{\prime}\right) \leq 3$. By Theorem 4.1.1, $\kappa^{\prime}\left(G^{\prime}\right)=2$. We apply induction on $n=\left|V\left(G^{\prime}\right)\right|$ to prove $G^{\prime} \in \mathcal{F}$.

Clearly, $n \geq 4$. If $n=4$, then $G=K_{2,2}$ and the result holds. We suppose that the result holds for fewer vertices.

Note that $\kappa^{\prime}\left(G^{\prime}\right)=2$. Let $X \subseteq E\left(G^{\prime}\right)$ be an edge cut of $G^{\prime}$ with $|X|=2$. Pick an $e \in X$, and denote $[e]=\left\{e^{\prime} \in E\left(G^{\prime}\right) \mid\left\{e, e^{\prime}\right\}\right.$ is an edge cut of $\left.G^{\prime}\right\} \cup\{e\}$. Then for any $\left\{e_{1}, e_{2}\right\} \subseteq[e],\left\{e_{1}, e_{2}\right\}$ is also an edge cut of $G$. Let $|[e]|=k \geq 2$. Then there are $k$ connected subgraphs $H_{1}, H_{2}, \cdots, H_{k}$ such that $H_{i}, H_{i+1}(i=1,2, \cdots, k-1)$ and $H_{1}, H_{k}$ are joined by one edge in $[e]$ (see Figure 4.2), and each $H_{i}(i=1,2, \cdots, k)$ is either a $K_{1}$ or 2-edge-connected.

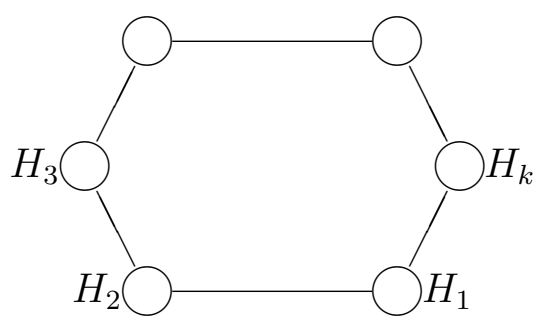

Figure 4.2 
Thus, we have

$$
\begin{aligned}
\sum_{i=1}^{k} F\left(H_{i}\right) & =\sum_{i=1}^{k}\left(2\left|V\left(H_{i}\right)\right|-\left|E\left(H_{i}\right)\right|-2\right) \\
& =2 \sum_{i=1}^{k}\left|V\left(H_{i}\right)\right|-\sum_{i=1}^{k}\left|E\left(H_{i}\right)\right|-2 k \\
& =2\left|V\left(G^{\prime}\right)\right|-\left(\left|E\left(G^{\prime}\right)\right|-k\right)-2 k=2\left|V\left(G^{\prime}\right)\right|-\left|E\left(G^{\prime}\right)\right|-k \\
& =F\left(G^{\prime}\right)-k+2 .
\end{aligned}
$$

We break it into four cases.

Case 1. $\quad k \geq 5$.

Note that $F\left(G^{\prime}\right) \leq 3$, we have $F\left(H_{i}\right)=0(i=1,2, \cdots, k)$ and $k=5$. Thus $H_{i}=K_{1}$ $(i=1,2, \cdots, k)$ and $G^{\prime}=C_{5}=C_{3}(1,1)$.

Case 2. $\quad k=4$.

Then $\sum_{i=1}^{4} F\left(H_{i}\right)=F\left(G^{\prime}\right)-2$. If $F\left(G^{\prime}\right) \leq 2$, then $F\left(H_{i}\right)=0(i=1,2,3,4)$ and $G^{\prime}=C_{4}=K_{2,2}$. If $F\left(G^{\prime}\right)=3$, then $\sum_{i=1}^{4} F\left(H_{i}\right)=1$. Without loss of generality, we assume that $F\left(H_{1}\right)=1$. By Theorem 2.0.5(ii), either $H_{1}$ is nontrivial and collapsible, contrary to the fact that $G^{\prime}$ is reduced, or $H_{1}=K_{2}$, contrary to the assumption that $\kappa^{\prime}\left(G^{\prime}\right) \geq 2$ or $k=4$.

Case 3. $\quad k=3$.

Then $\sum_{i=1}^{3} F\left(H_{i}\right)=F\left(G^{\prime}\right)-1$. Note that a triangle is collapsible, we have $F\left(G^{\prime}\right)=3$, and there doesn't exist some $H_{i}$ such that $F\left(H_{i}\right)=1$. Without loss of generality, we let $F\left(H_{1}\right)=2$ and $F\left(H_{2}\right)=F\left(H_{3}\right)=0$. Then $H_{2}=H_{3}=K_{1}$. Note that $H_{1}$ is 2-edgeconnected, we have $H_{1}=K_{2, t}(t \geq 2)$ by Theorem 2.0.5(iii). Thus $G^{\prime}$ must be one of the following graphs shown in Figure 4.3. 


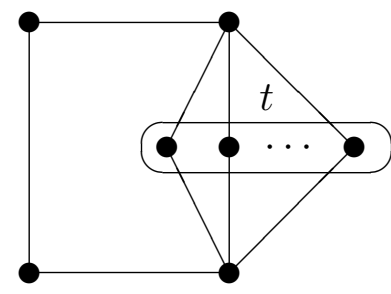

$G^{\prime}=C_{3}(t, 1)$

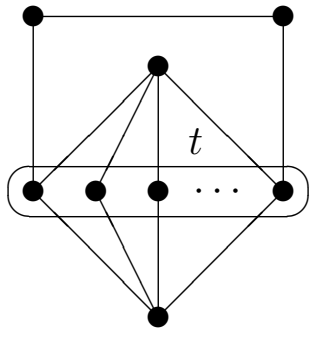

$G^{\prime}=C_{3}(2,1)($ when $t=2)$

$G^{\prime}=K_{2,3}(1, t-2)($ when $t \geq 3)$

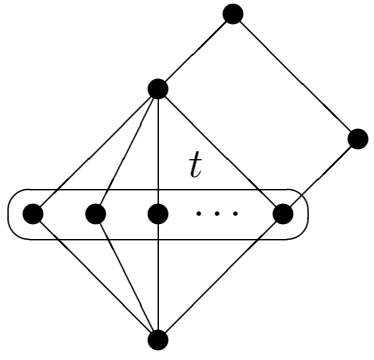

$G^{\prime}=S(t-1,1)$

Figure 4.3

Case $4 k=2$.

Let $H_{1}, H_{2}$ be the two components of $G^{\prime}-[e]$ and we assume that $F\left(H_{1}\right) \leq F\left(H_{2}\right)$. Since $\sum_{i=1}^{2} F\left(H_{i}\right)=F\left(G^{\prime}\right) \leq 3, F\left(H_{1}\right) \leq 1$. Note that for $i=1,2$, either $H_{i}=K_{1}$ or $H_{i}$ is 2-edge-connected, we have $H_{1}=K_{1}$ by Theorem 2.0.5(ii), and $H_{2} \neq K_{1}$ since $C_{2}$ is collapsible. Therefore $F\left(H_{2}\right) \leq 3$ and $\kappa^{\prime}\left(H_{2}\right) \geq 2$.

Note that $\mathrm{H}_{2}$ is reduced, we have $\mathrm{H}_{2} \in \mathcal{F}$ by induction. Thus there exists a vertex $v \in V\left(G^{\prime}\right)$ such that $d_{G^{\prime}}(v)=2$ and $v$ is one vertex of a 4-cycle of $G^{\prime}$. Let $G_{1}=G^{\prime}-v$. Then $G_{1} \in \mathcal{F}$ by induction.

When $G_{1}=K_{4}\left(s_{1}, s_{2}, s_{3}\right)$, there are 4 possible way for $v$ to join $G_{1}$ (see Figure 4.4 ). Let $\left\{s_{i}, s_{j}, s_{k}\right\}=\left\{s_{1}, s_{2}, s_{3}\right\}$. Then for the first graph in Figure 4.4, in which case $G^{\prime}=$ $K_{4}\left(s_{i}+1, s_{j}, s_{k}\right)$. By Lemma 2.0.8 and Theorem 2.0.7(iii), each of the other graphs in Figure 4.4 contains a collapsible graph, and so $G^{\prime}$ could not be these three graphs.
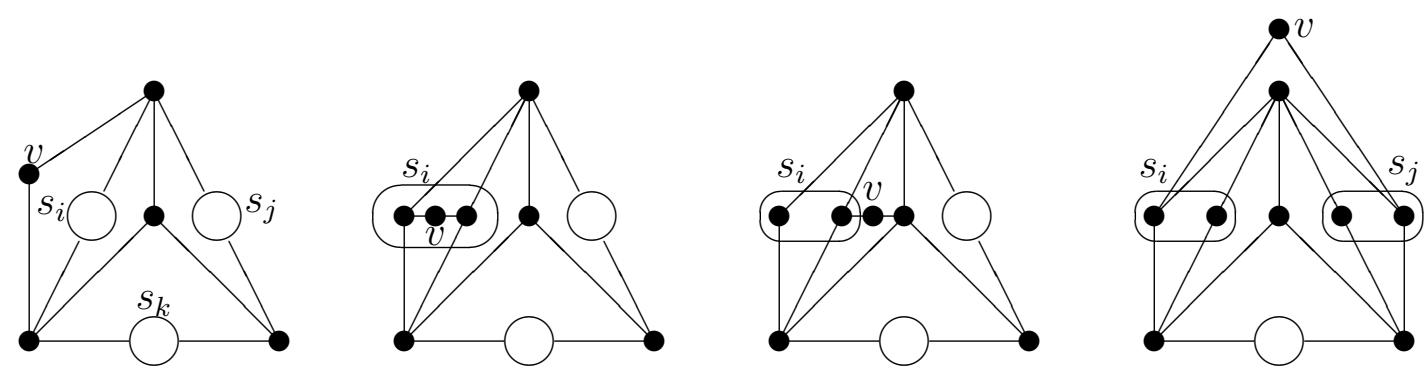

Figure 4.4 
Similarly, we can check other 5 cases. If $G_{1}=K_{2, t}(t \geq 2)$, then $G^{\prime}=K_{2, t+1}$. If $G_{1}=T\left(s_{1}, s_{2}\right)$, then $G^{\prime} \in\left\{T\left(s_{1}+1, s_{2}\right), T\left(s_{1}, s_{2}+1\right), K_{4}\left(s_{1}, s_{2}, 1\right), S\left(2, s_{1}\right), S\left(2, s_{2}\right)\right\}$. If $G_{1}=C_{3}\left(s_{1}, s_{2}\right)$, then $G^{\prime} \in\left\{C_{3}\left(s_{1}+1, s_{2}\right), C_{3}\left(s_{1}, s_{2}+1\right), K_{2,3}\left(s_{1}, 1\right), K_{2,3}\left(s_{2}, 1\right)\right\}$. If $G_{1}=$ $S\left(s_{1}, s_{2}\right)$, then $G^{\prime} \in\left\{S\left(s_{1}+1, s_{2}\right), S\left(s_{1}, s_{2}+1\right), T\left(2, s_{1}\right), T\left(2, s_{2}\right), K_{4}\left(1,1, s_{1}\right), K_{4}\left(1,1, s_{2}\right)\right\}$. If $G_{1}=K_{2,3}\left(s_{1}, s_{2}\right)$, then $G^{\prime} \in\left\{K_{2,3}\left(s_{1}+1, s_{2}\right), K_{2,3}\left(s_{1}, s_{2}+1\right)\right\}$.

\subsection{Proofs of Theorems 4.1.3 and 4.1.4}

The following theorem and lemma are needed in the proof of Theorem 4.1.3.

Theorem 4.3.1 (Chen et al., [13]) If $G$ is a 3-edge-connected planar graph with $|V(G)| \leq$ 23 , then $G$ is supereulerian.

Lemma 4.3.2 Let $C=v_{1} v_{2} v_{3} v_{4} v_{1}$ be a cycle of a graph $G$ with $N\left(v_{i}\right)-V(C)=\left\{u_{i}\right\}$ $(i=1,2,3,4)$, and with either $u_{1} \neq u_{2}$ or $u_{1} \neq u_{3}$. If $a_{1}(G) \leq 2$, then $a_{1}\left(G_{\pi}\right) \leq 2$.

Proof. Let $\left(E_{1}, E_{2}\right)$ be a partition of $E(G)$ such that each $G\left[E_{i}\right](i=1,2)$ is acyclic, and let

$$
\begin{aligned}
& E=E(C) \cup\left\{u_{1} v_{1}, u_{2} v_{2}, u_{3} v_{3}, u_{4} v_{4}\right\}, E_{1}^{\prime}=E_{1}-E, E_{2}^{\prime}=E_{2}-E \\
& E_{11}=E_{1}^{\prime} \cup\left\{u_{1} v_{1}, u_{4} v_{4}, v_{1} v_{2}, v_{2} v_{3}\right\}, \quad E_{12}=E_{2}^{\prime} \cup\left\{u_{2} v_{2}, u_{3} v_{3}, v_{3} v_{4}, v_{4} v_{1}\right\} \\
& E_{21}=E_{1}^{\prime} \cup\left\{u_{1} v_{1}, u_{2} v_{2}, v_{2} v_{3}, v_{3} v_{4}\right\}, \quad E_{22}=E_{2}^{\prime} \cup\left\{u_{3} v_{3}, u_{4} v_{4}, v_{1} v_{2}, v_{4} v_{1}\right\} \\
& E_{31}=E_{1}^{\prime} \cup\left\{u_{2} v_{2}, u_{3} v_{3}, v_{3} v_{4}, v_{4} v_{1}\right\}, \quad E_{32}=E_{2}^{\prime} \cup\left\{u_{1} v_{1}, u_{4} v_{4}, v_{1} v_{2}, v_{2} v_{3}\right\} \\
& E_{41}=E_{1}^{\prime} \cup\left\{u_{3} v_{3}, u_{4} v_{4}, v_{1} v_{2}, v_{4} v_{1}\right\}, \quad E_{42}=E_{2}^{\prime} \cup\left\{u_{1} v_{1}, u_{2} v_{2}, v_{2} v_{3}, v_{3} v_{4}\right\}
\end{aligned}
$$

Then for each $i=1,2,3,4,\left(E_{i 1}, E_{i 2}\right)$ is also a partition of $E(G)$ such that each $G\left[E_{i j}\right]$ $(j=1,2)$ is acyclic. Let $e_{\pi}=w_{1} w_{2}$ be the new edge in $G_{\pi}$, and for $i=1,2,3,4$, let

$$
E_{i 1}^{\prime}=E_{i 1}-E(C), \quad E_{i 2}^{\prime}=\left(E_{i 2}-E(C)\right) \cup\left\{e_{\pi}\right\}
$$

Then $\left(E_{i 1}^{\prime}, E_{i 2}^{\prime}\right)$ is a partition of $E\left(G_{\pi}\right)$. Suppose that $a_{1}\left(G_{\pi}\right) \geq 3$. Note that $G_{\pi}\left[E_{i 1}^{\prime}\right]$ is acyclic by the construction of $\left(E_{i 1}, E_{i 2}\right), G_{\pi}\left[E_{12}^{\prime}\right], G_{\pi}\left[E_{22}^{\prime}\right], G_{\pi}\left[E_{32}^{\prime}\right], G_{\pi}\left[E_{42}^{\prime}\right]$ contain 
cycles $u_{2} P_{1} u_{3} w_{1} w_{2} u_{2}, u_{3} P_{2} u_{4} w_{2} w_{1} u_{3}, u_{4} P_{3} u_{1} w_{1} w_{2} u_{4}, u_{1} P_{4} u_{2} w_{2} w_{1} u_{1}$, respectively, where $P_{1}, P_{2}, P_{3}, P_{4}$ respectively are $\left(u_{2}, u_{3}\right)$-path, $\left(u_{3}, u_{4}\right)$-path, $\left(u_{4}, u_{1}\right)$-path and $\left(u_{1}, u_{2}\right)$-path in $G$. As either $u_{1} \neq u_{2}$ or $u_{1} \neq u_{3}, G\left[E_{2}\right]$ contains a cycle $C \subseteq P_{1} \cup P_{2} \cup P_{3} \cup P_{4}$, a contradiction.

Proof of Theorem 4.1.3. By contradiction, suppose that $G$ is a smallest counterexample. Then $G$ is reduced.

Claim 1. $\kappa^{\prime}(G) \geq 3$.

Since $\kappa_{e}(G) \geq 3$, that is, $G$ is essentially 3 -edge-connected, we only need to prove that $d_{G}(v) \geq 3$ for any $v \in V(G)$. Suppose that there exists $v \in V(G)$ such that $d_{G}(v)=2$. Let $e_{1}=v u_{1}, e_{2}=v u_{2}$. Note that $G$ is reduced, $G$ doesn't contain triangle. Thus $u_{1} u_{2} \notin E(G)$. Let $G_{1}=G / e_{1}$. Then $\kappa^{\prime}\left(G_{1}\right) \geq 2, \kappa_{e}\left(G_{1}\right) \geq 3$ and $F\left(G_{1}\right) \leq 5$. Note that $G$ is smallest, there exists a dominating Eulerian subgraph $H^{\prime}$ in $G_{1}$ such that $D_{3}^{*}\left(G_{1}\right) \subseteq V\left(H^{\prime}\right)$. By $u_{1} u_{2} \notin E(G)$ and $\kappa_{e}(G) \geq 3$, we have $d_{G}\left(u_{1}\right) \geq 3$ and $d_{G}\left(u_{2}\right) \geq 3$. Thus $d_{G_{1}}\left(u_{i}\right) \geq 3(i=1,2)$ and $u_{1}, u_{2} \in H$. Let $H=\left\{\begin{array}{ll}H^{\prime}, & \text { if } u_{1} u_{1} \notin E\left(H^{\prime}\right) \\ G\left[\left(E\left(H^{\prime}\right)-\left\{u_{1} u_{2}\right\}\right) \cup\left\{v u_{1}, v u_{2}\right\}\right], & \text { if } u_{1} u_{2} \in E\left(H^{\prime}\right)\end{array}\right.$. Then $H$ is a dominating Eulerian subgraph of $G$ such that $D_{3}^{*}(G) \subseteq H$, a contradiction.

Claim 2. $\kappa_{e}(G) \geq 4$.

Suppose that $S$ is a 3 -edge cut and $G_{1}, G_{2}$ are two components of $G-S$ with $F\left(G_{1}\right) \leq$ $F\left(G_{2}\right)$ and $E\left(G_{1}\right) \neq \emptyset, E\left(G_{2}\right) \neq \emptyset$. Then $F\left(G_{1}\right)+F\left(G_{2}\right)=F(G)+1 \leq 6$ by Theorem 2.0.7(iv). Thus we have $F\left(G_{1}\right) \leq 3$.

If $G_{1}$ has an cut edge $e$, let $H_{1}$ and $H_{2}$ be two components of $G_{1}-e$ and $H_{2}$ be the component adjacent to at least two edges of $S$. Then either $\left[V\left(H_{1}\right), V(G)-V\left(H_{1}\right)\right]_{G}=e$ or $\left[V\left(H_{1}\right), V(G)-V\left(H_{1}\right)\right]_{G}$ is a 2-edge cut in $G$, contrary to Claim 1 . So we have $\kappa^{\prime}\left(G_{1}\right) \geq 2$. Note that $G_{1}$ is reduced and $\left|V\left(G_{1}\right)\right| \geq 2$, by Theorem 4.1.2, $G_{1} \in \mathcal{F}$. Since $\left|D_{2}\left(G_{1}\right)\right| \leq 3$ and by planarness of $G, G_{1}=K_{4}(1,1,1)$. Similarly, $G_{2}=K_{4}(1,1,1)$. So $G$ must be the graph shown in Figure 4.5. Clearly, $G$ is supereulerian, a contradiction. 


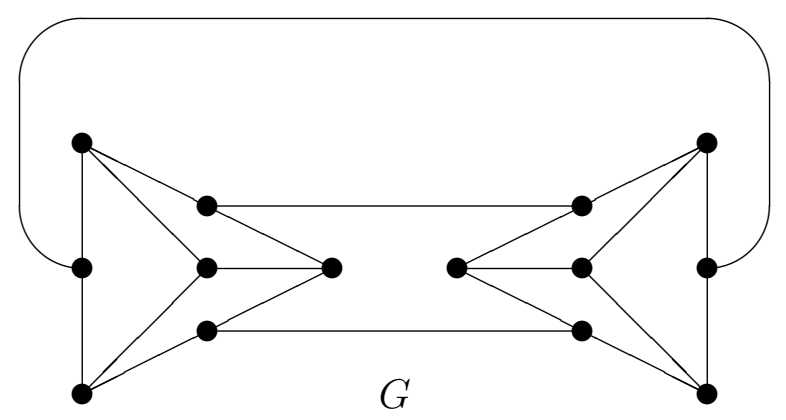

Figure 4.5

Claim 3. $G$ has at least 134 -faces.

By Claim 1 and Theorem 4.3.1, we have $n=|V(G)| \geq 24$. Let $x$ be the number of 4-faces, $m$ the number of the edges, $f$ the number of faces. Then $2 m \geq 4 x+5(f-x)$. Thus $2 m \geq 5 f-x$. Note that $n-m+f=2$, we have $5 n-3 m \geq 10-x$. Since $F(G)=2 n-m-2 \leq 5$, we have $x \geq n-11 \geq 13$. Thus $G$ has at least 134 -faces.

Claim 4. No two 4-faces $C_{1}=v_{1} v_{2} v_{3} v_{4} v_{1}$ and $C_{2}=v_{1} v_{2} v_{5} v_{6} v_{1}$ in $G$ satisfy $d_{G}\left(v_{i}\right)=$ $3(i=1,2 \cdots, 6)$.

By contradiction. Suppose that there exist two 4-faces $C_{1}=v_{1} v_{2} v_{3} v_{4} v_{1}$ and $C_{2}=$ $v_{1} v_{2} v_{5} v_{6} v_{1}$ in $G$ such that $d_{G}\left(v_{i}\right)=3(i=1,2 \cdots, 6)$. Let $H=G-\left\{v_{1}, v_{2}, \cdots, v_{6}\right\}$. Then we can get the new graph $\left(G_{\pi}\right)_{\pi}$ by using $\pi$-collapsible 2 times (see the graphs in Figure 4.6).

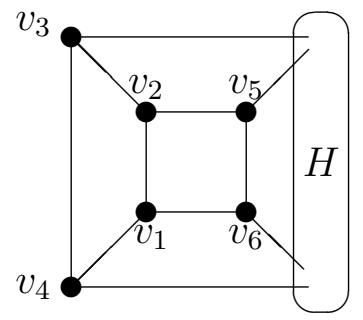

G

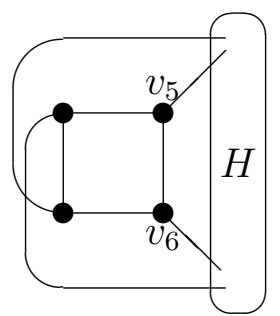

$G_{\pi}$

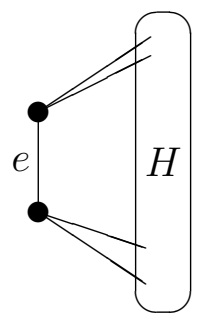

$\left(G_{\pi}\right) / \pi$

Figure 4.6 
Let $e=w_{1} w_{2}$ denote the new edge in $\left(G_{\pi}\right)_{\pi}$. Clearly, $\left(G_{\pi}\right)_{\pi}$ is 2-edge-connected, otherwise, $e$ is a cut edge of $\left(G_{\pi}\right)_{\pi}$ and $X=\left\{v_{1} v_{2}, v_{3} v_{4}, v_{5} v_{6}\right\}$ is a 3-edge cut in $G$ in which both sides of $G-X$ have edges, contrary to Claim 2. Next we want to prove that $G$ is 3 -edge-connected. Suppose that $G$ is not 3 -edge-connected. Then $\left\{e, e_{1}\right\}$ is a 2-edge cut, where $e_{1}=w_{3} w_{4} \in E(H)$. Let $H_{1}, H_{2}$ be two components of $\left(G_{\pi}\right)_{\pi}-\left\{e, e_{1}\right\}$, and $w_{1}, w_{3} \in V\left(H_{1}\right), w_{2}, w_{4} \in V\left(H_{2}\right)$. Then $E\left(H_{1}-\left\{w_{1}\right\}\right)=\emptyset$ and $E\left(H_{2}-\left\{w_{2}\right\}\right)=\emptyset$ by Claim 2. Thus $N_{\left(G_{\pi}\right)_{\pi}}\left(w_{1}\right)=w_{3}$ and $N_{\left(G_{\pi}\right)_{\pi}}\left(w_{2}\right)=w_{4}$. Hence $V(H)=\left\{w_{3}, w_{4}\right\}$, $N_{G}\left(v_{3}\right) \cap H=N_{G}\left(v_{5}\right) \cap H=\left\{w_{3}\right\}$ and $N_{G}\left(v_{6}\right) \cap H=N_{G}\left(v_{4}\right) \cap H=\left\{w_{4}\right\}$. So $G$ is supereulerian, a contradiction. Thus $G$ is 3-edge-connected. Clearly, $\left(G_{\pi}\right)_{\pi}$ is planar with $F\left(\left(G_{\pi}\right)_{\pi}\right)=F(G)-2 \leq 3$ by Lemma 4.3 .2 and Theorem 2.0.7(iv). By Theorem 4.1.2, $\left(G_{\pi}\right)_{\pi}$ is supereulerian. Thus $G$ is supereulerian by Theorem 2.0.7(i), a contradiction. So Claim 4 holds.

Claim 5. Suppose that $C=v_{1} v_{2} v_{3} v_{4} v_{1}$ is a 4 -face of $G$. Then for $i=1,2,3,4$, $d_{G}\left(v_{i}\right)=3$.

Let $G_{1}=\left(G-\left\{v_{1} v_{2}, v_{3} v_{4}\right\}\right) /\left\{v_{1} v_{4}, v_{2} v_{3}\right\}$ and $G_{2}=\left(G-\left\{v_{1} v_{4}, v_{2} v_{3}\right\}\right) /\left\{v_{1} v_{2}, v_{3} v_{4}\right\}$. First we prove that either $\kappa_{e}\left(G_{1}\right) \geq 3$ or $\kappa_{e}\left(G_{2}\right) \geq 3$. Suppose that $\kappa_{e}\left(G_{i}\right)=2$ for $i=1,2$. Then $G$ must have the structure in Figure 4.7 .

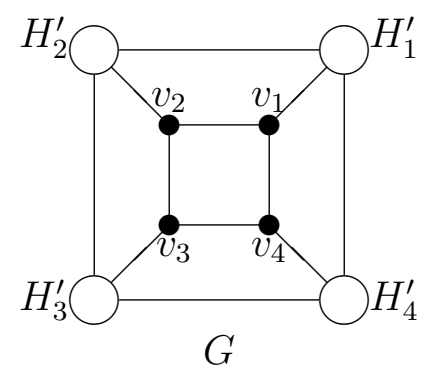

Figure 4.7 
For $i=1,2,3,\left|\left[V\left(H_{i}^{\prime}\right), V\left(H_{i+1}^{\prime}\right)\right]_{G}\right|=1$ and $\left|\left[V\left(H_{4}^{\prime}\right), V\left(H_{1}^{\prime}\right)\right]_{G}\right|=1$. Let $H_{i}=H_{1}^{\prime} \cup\left\{v_{i}\right\}$ $(i=1,2,3,4)$. Then

$$
\begin{aligned}
\sum_{i=1}^{4} F\left(H_{i}\right) & =\sum_{i=1}^{4}\left(2\left|V\left(H_{i}\right)\right|-\left|E\left(H_{i}\right)\right|-2\right) \\
& =2 n-(m-8)-8=2 n-m=F(G)+2 \leq 7 .
\end{aligned}
$$

Note that $\left|V\left(H_{i}\right)\right| \geq 2$, there are at least three of these $H_{i}$ 's, say $H_{1}, H_{2}, H_{3}$, such that $F\left(H_{i}\right) \leq 2(i=1,2,3)$. Since $\kappa^{\prime}(G) \geq 3$, we have $H_{i} \neq K_{2, t}(i=1,2,3)$. Thus $H_{1}=H_{2}=H_{3}=K_{2}$ by Theorem 2.0.5(iii). This contradicts Claim 4. So without loss generality, we assume that $\kappa_{e}\left(G_{1}\right) \geq 3$ and $w_{1}, w_{2}$ are two new vertices. By the assumption of $G, G_{1}$ has a dominating Eulerian subgraph $H^{\prime}$ such that $D_{3}^{*}\left(G_{1}\right) \subseteq H^{\prime}$. If either $w_{1} \in H^{\prime}$ or $w_{2} \in H^{\prime}$, then we can always get a dominating Eulerian subgraph $H$ of $G$ such that $D_{3}^{*}(G) \subseteq H$, it is impossible. Thus $w_{1}, w_{2} \notin H^{\prime}$. Therefore Claim 5 holds.

By Claim 3, let $C_{1}, C_{2}, C_{3}, C_{4}, C_{5}$ be five 4-faces of $G$. By Claims 4,5, no two of these 4faces have common vertices or edges. Applying $\pi$-collapsible to each of these five 4 -faces, we get the graph $G_{3}$ with $F\left(G_{3}\right)=0$ by Lemma 4.3 .2 and Theorem 2.0.7(iv). Note that $G_{3}$ is connected, $G_{3}$ is supereulerian by Theorem 2.0.5(i). Thus $G$ is also supereulerian, a contradiction.

Proof of Theorem 4.1.4. Let $G^{\prime}$ be the reduction of $G$. If $G^{\prime}=K_{1}$, then $G$ is supereulerian. Next we suppose that $G^{\prime} \neq K_{1}$. Then $G^{\prime}$ is 3-edge-connected and nontrivial. Denote $d_{i}=\left|D_{i}\left(G^{\prime}\right)\right|(i \geq 3)$.

If $d_{3} \geq 17$, then we assume that $v_{1}, v_{2}, \cdots, v_{17}$ are the vertices of $V\left(G^{\prime}\right)$ in $D_{3}\left(G^{\prime}\right)$, i.e. $d_{G^{\prime}}\left(v_{i}\right)=3$ for each $i$, and the corresponding pre-images are $H_{1}, H_{2}, \cdots, H_{17}$. Each $H_{i}$ is joined to the rest of $G$ by an edge cut consisting of $d_{G^{\prime}}\left(v_{i}\right)=3$ edges. By the hypothesis of Theorem 4.1.4, $\left|V\left(H_{i}\right)\right| \geq \frac{n}{16}$, and

$$
n=|V(G)| \geq \sum_{i=1}^{17}\left|V\left(H_{i}\right)\right| \geq \frac{17 n}{16},
$$

a contradiction. So $d_{3} \leq 16$. 
By Theorem 4.1.3, we only need to consider $F\left(G^{\prime}\right) \geq 6$. Note that $\left|V\left(G^{\prime}\right)\right|=\sum_{i \geq 3} d_{i}$, $2\left|E\left(G^{\prime}\right)\right|=\sum_{v \in V(G)} d_{G}(v)=\sum_{i \geq 3} i d_{i}$, and $F\left(G^{\prime}\right)=2\left|V\left(G^{\prime}\right)\right|-\left|E\left(G^{\prime}\right)\right|-2$, we have the following

$$
d_{3} \geq 16+\sum_{i \geq 5}(i-4) d_{i}
$$

Thus $n=d_{3}=16$. By Theorem 4.3.1, $G$ is supereulerian.

\subsection{Proofs of Theorems 4.1.7 and 4.1.8}

We shall apply Theorem 4.1.1 to prove both Theorems 4.1.7 and 4.1.8. First, we need one more lemma in this section.

Lemma 4.4.1 Let $G$ be a planar graph such that every face of $G$ has degree at least 4. Then $|E(G)| \leq 2|V(G)|-4$.

Proof. Let $f$ denote the number of faces of $G$. Since every face of $G$ has degree at least $4,4 f \leq 2|E(G)|$ and so the lemma follows from Euler's formula.

Proof of Theorem 4.1.7. Let $G$ be a planar graph with $\kappa^{\prime}(G) \geq 3$ and with $n$ vertices. Assume that $|E(G)| \geq 2 n-5$. Let $G^{\prime}$ denote the reduction of $G$. By Theorem 2.0.4(iv), it suffices to show $G^{\prime}=K_{1}$.

By contradiction, assume that $G^{\prime}$ is nontrivial. Then by Theorem 2.0.4(ii), $G^{\prime}$ also has girth at least 4 . Note that $G^{\prime}$ is planar and $\kappa^{\prime}\left(G^{\prime}\right) \geq 3$, let $H_{1}, \cdots H_{l}$ denote the nontrivial maximal collapsible subgraphs of $G$ and let $p$ denote the number of vertices of $G^{\prime}$. Then by Lemma 4.4.1, each $\left|E\left(H_{i}\right)\right| \leq 2\left|V\left(H_{i}\right)\right|-4$,

$$
2 n-5 \leq|E(G)|=\sum_{i=1}^{l}\left|E\left(H_{i}\right)\right|+\left|E\left(G^{\prime}\right)\right| \leq 2 \sum_{i=1}^{l}\left|V\left(H_{i}\right)\right|-4 l+\left|E\left(G^{\prime}\right)\right|,
$$

and so $\left|E\left(G^{\prime}\right)\right| \geq 2 p-5+2 l$. By Theorem 2.0.5(iii) and $\kappa^{\prime}\left(G^{\prime}\right) \geq 3$, we have $F\left(G^{\prime}\right) \geq 3$. Thus, by Theorem 2.0.7(iv), $2 p-5 \geq\left|E\left(G^{\prime}\right)\right| \geq 2 p-5+2 l$, and so $l=0$ and $F\left(G^{\prime}\right)=3$. 
Therefore by Theorem 4.1.1, $G^{\prime}$ must be collapsible, contrary to the fact that $G^{\prime}$ does not have nontrivial collapsible subgraphs. This proves Theorem 4.1.7.

Proof of Theorem 4.1.8. Let $G^{\prime}$ be the reduction of $G$. Again we argue by contradiction and assume that $G^{\prime} \neq K_{1}$. Note that $\kappa^{\prime}\left(G^{\prime}\right) \geq 3$, by Euler's formula, by Theorem 2.0.7(iv) and by (4.1) in the process of the proof of Theorem 4.1.1, one concludes that $F\left(G^{\prime}\right)=3$ and so Theorem 4.1.8 follows from Theorem 4.1.1.

\subsection{Proofs of Theorems 4.1.9 and 4.1.10}

A few more lemmas and a former theorem of Nash-Williams and Tutte are needed in the proofs in this section.

Theorem 4.5.1 (Nash-Williams [21] and Tutte [26]) A graph $G=(V, E)$ contains $l$ edge-disjoint spanning trees if and only if for each partition $\left(V_{1}, V_{2}, \cdots, V_{k}\right)$ of $V$, the number of edges which have end in different parts of the partition is at least $l(k-1)$.

Proof of Theorem 4.1.9. Suppose $V=\left(V_{1}, V_{2}, \cdots, V_{k}\right)$ is any partition of $V$. Without loss of generality, let $\left|V_{1}\right|=\left|V_{2}\right|=\cdots=\left|V_{l}\right|=1, \quad\left|V_{l+1}\right|=\cdots=\left|V_{l+m}\right|=2$, and for $l+m+1 \leq j \leq k,\left|V_{j}\right| \geq 3$. Since $G$ is a 2-connected simple planar graph, $\left|E\left(G\left[V_{j}\right]\right)\right| \leq 3\left|V_{j}\right|-6$ for $l+m+1 \leq j \leq k ;\left|E\left(G\left[V_{i}\right]\right)\right| \leq 1$ for $l+1 \leq i \leq l+m$; and $E\left(G\left[V_{j}\right]\right)=\emptyset$ for $1 \leq j \leq l$. Then, we have

$$
\begin{aligned}
\sum_{1 \leq i<j \leq k}\left|\left[V_{i}, V_{j}\right]_{G}\right| & =|E(G)|-\sum_{j=1}^{k}\left|E\left(G\left[V_{j}\right]\right)\right| \\
& \geq 3 n-8-m-[3(n-2 m-l)-6(k-l-m)] \\
& =6 k-3 l-m-8 \\
& =2(k-1)+4 k-3 l-m-6 .
\end{aligned}
$$

We consider the following cases. 
Case 1. $l=0$.

By $n \geq 6$ and $k \geq 2,4 k-3 l-m-6=3 k+(k-m)-6 \geq 0$.

Case 2. $m=0$.

If $l=k$, then $4 k-3 l-m-6=k-6 \geq 0$ since $k=n \geq 6$. If $l<k$, then $4 k-3 l-m-6=$ $3(k-l)+k-6 \geq 0$ except for $l=1, k=2$. But when $l=1, k=2$, since $G$ is a 2-edge-connected simple graph, $\left|\left[V_{1}, V_{2}\right]_{G}\right| \geq 2=2(k-1)$.

Case 3. $l>0$ and $m>0$.

If $k=l+m$, then $4 k-3 l-m-6=3 m+l-6=(2 m+l)+m-6=n+m-6 \geq 0$ since $n=2 m+l \geq 6$. If $k>l+m$, then $k-l \geq m+1 \geq 2$ and $k-m \geq 0$. It follows that $4 k-3 l-m-6=3(k-l)+(k-m)-6 \geq 0$.

Therefore, in any case, $\sum_{1 \leq i<j \leq k}\left|\left[V_{i}, V_{j}\right]_{G}\right| \geq 2(k-1)$, and so $G$ must have two edgedisjoint spanning trees by Theorem 4.5.1.

We shall prove a stronger result than Theorem 4.1.10, as stated below.

Theorem 4.5.2 If $G$ is a 2-edge-connected simple planar graph with $n \geq 9$ vertices and with $|E(G)| \geq 3 n-12$ edges, then exactly one of the following holds:

(i) $G$ is collapsible.

(ii) The reduction of $G$ is a 4-cycle.

(iii) The reduction of $G$ is isomorphic to $K_{2,3}$ with exactly one nontrivial vertex whose pre-image is a maximal planar graph of $n-4$ vertices.

We need two more lemmas.

Lemma 4.5.3 If $G$ is a simple planar graph with $n \geq 9$ vertices and with $|E(G)| \geq$ $3 n-12$, then $G$ is not reduced.

Proof. If $G$ is reduced, then by Theorem 2.0.4(ii) and Lemma 4.4.1, $2 n-4 \geq|E(G)| \geq$ $3 n-12$, whence $n \leq 8$, contrary to the assumption that $n \geq 9$. Therefore, $G$ is not reduced. 
Lemma 4.5.4 Let $C>0$ be a constant, and let $G$ be a simple planar graph with $n$ vertices and with $l$ nontrivial maximal collapsible subgraphs. Let $G^{\prime}$ denote the reduction of $G$. If $|E(G)| \geq 3 n-C$, then

$$
\left|E\left(G^{\prime}\right)\right| \geq 3\left|V\left(G^{\prime}\right)\right|-C+3 l
$$

Proof. Let $H_{1}, \cdots H_{l}$ be nontrivial maximal collapsible subgraphs of $G$. Let $G^{\prime}=$ $G /\left(\bigcup_{i=1}^{l} E\left(H_{i}\right)\right)$ be the reduction of $G$ with $n^{\prime}$ vertices. Since each $H_{i}$ is a nontrivial planar graph for each $i$ with $1 \leq i \leq l$,

$$
\left|E\left(H_{i}\right)\right| \leq 3\left|V\left(H_{i}\right)\right|-6
$$

Note that $\left|V\left(G^{\prime}\right)\right|=n-\sum_{i=1}^{l}\left|V\left(H_{i}\right)\right|+l$. It follows from (4.6) that

$$
\begin{aligned}
\left|E\left(G^{\prime}\right)\right| & =|E(G)|-\sum_{i=1}^{l}\left|E\left(H_{i}\right)\right| \geq|E(G)|-\sum_{i=1}^{l}\left(3\left|V\left(H_{i}\right)\right|-6\right) \\
& \geq 3 n-C-3\left(n-\left|V\left(G^{\prime}\right)\right|+l\right)+6 l=3\left|V\left(G^{\prime}\right)\right|-C+3 l .
\end{aligned}
$$

This proves the lemma.

Proof of Theorem 4.5.2. Since the 4-cycle and $K_{2,3}$ are not collapsible, (i),(ii) and (iii) are mutually exclusive. We assume that both Theorem 4.5.2(i) and Theorem 4.5.2(ii) are false, and want to prove that Theorem 4.5.2(iii) must hold.

By Lemma 4.5.3, $G$ is not reduced. Let $H_{1}, \cdots H_{l}$ be the nontrivial maximal collapsible subgraphs of $G$. Let $G^{\prime}=G /\left(\bigcup_{i=1}^{l} E\left(H_{i}\right)\right)$ be the reduction of $G$ with $n^{\prime}$ vertices. By Theorem 2.0.4(iii), $G^{\prime}$ is reduced and so by Lemma 4.5.4 with $C=12$ and $l \geq 1$, we have

$$
\left|E\left(G^{\prime}\right)\right| \geq 3\left|V\left(G^{\prime}\right)\right|-12+3 l .
$$

By Theorem 2.0.4(ii) and Lemma 4.4.1,

$$
2\left|V\left(G^{\prime}\right)\right|-4 \geq\left|E\left(G^{\prime}\right)\right|
$$

It follows from (4.7) and (4.8) that

$$
2\left|V\left(G^{\prime}\right)\right|-4 \geq\left|E\left(G^{\prime}\right)\right| \geq 3\left|V\left(G^{\prime}\right)\right|-12+3 l .
$$


By (4.9), $\left|V\left(G^{\prime}\right)\right| \leq 8-3 l$. Since any 2-edge-connected graph with 3 vertices is collapsible, and since any simple graph with 4 vertices is either collapsible or is isomorphic to the 4-cycle, we must have $l=1$ and $\left|V\left(G^{\prime}\right)\right|=5$. Therefore, equalities must hold everywhere in (4.9), and so by Theorem 2.0.5(iii) and by $\left|V\left(G^{\prime}\right)\right|=5, G^{\prime} \cong K_{2,3}$ with exactly one nontrivial vertex, named by $H$.

Note that $|E(H)| \geq|E(G)|-6 \geq 3 n-18$ and that $|V(H)|=n-4$. It follows that $|E(H)| \geq 3(n-4)-6=3|V(H)|-6$, and so $H$ must be a maximal planar graph with $n-4$ vertices.

Proof of Theorem 4.1.10. Note that 4-cycles are supereulerian, and so Theorem 4.1.9 follows from Theorem 2.0.4(iv) and Theorem 4.5.2. 


\section{Chapter 5}

\section{Hamiltonicity in 3-connected claw-free graphs}

\subsection{Introduction}

Let $H$ be the line graph $L(G)$ of a graph $G$. Then the order $\nu(H)$ of $H$ is equal to the number $m(G)$ of edges of $G$, and $\delta(H)=\min \left\{d_{G}(x)+d_{G}(y)-2 \mid x y \in E(G)\right\}$. If $L(G)$ is $k$-connected, then $G$ is essentially $k$-edge-connected, which means that the only edge-cut sets of $G$ having less than $k$ edges are the sets of edges incident with some vertex of $G$.

A graph $H$ is claw-free if it does not contain $K_{1,3}$ as an induced subgraph. In [25], Ryjáček defined the closure $\operatorname{cl}(H)$ of a claw-free graph $H$ to be one obtained by recursively adding edges to join two nonadjacent vertices in the neighborhood of any locally connected vertex of $H$, as long as this is possible.

Theorem 5.1.1 (Ryjáček, [25]) Let $H$ be a claw-free graph and $c l(H)$ its closure. Then (i) $\operatorname{cl}(H)$ is well-defined, and $\kappa(\operatorname{cl}(H)) \geq \kappa(H)$,

(ii) there is triangle-free graph $G$ such that $c l(H)=L(G)$,

(iii) both graphs $H$ and $c l(H)$ have the same circumference. 
As a corollary of Theorem 5.1.1, a claw-free graph $H$ is hamiltonian if and only if $\operatorname{cl}(H)$ is hamiltonian, and so $H$ is said to be closed if $H=\operatorname{cl}(H)$.

Many works have been done to give sufficient conditions for a claw-free graph $H$ to be hamiltonian in terms of its minimum degree $\delta(H)$. These conditions depend on the connectivity $\kappa(H)$. If $\kappa(H)=4$, Matthews and Sumner [22] conjectured that $H$ is hamiltonian and this conjecture is still open. When $\kappa(H)=2$, Kuipers and Veldman [18], and independently Favaron, Flandrin, Li and Ryjáček [14], proved that if $H$ is a 2-connected claw-free graph with sufficiently large order $\nu$, and if $\delta(H) \geq \frac{\nu+c}{6}$ (where $c$ is a constant), then $H$ is hamiltonian except a member of ten well-defined families of graphs. When $\kappa(H)=3$, the following have been proved and proposed.

Theorem 5.1.2 (Kuipers and Veldman, [18]) If $H$ is a 3-connected claw-free simple graph with sufficiently large order $\nu$, and if $\delta(H) \geq \frac{\nu+29}{8}$, then $H$ is hamiltonian.

Theorem 5.1.3 (Favaron and Fraisse, [15]) If $H$ is a 3-connected claw-free simple graph with order $\nu$, and if $\delta(H) \geq \frac{\nu+37}{10}$, then $H$ is hamiltonian.

Conjecture 5.1.4 (Kuipers and Veldman, [18], see also [15]) Let $H$ be a 3-connected claw-free simple graph of order $\nu$ with $\delta(H) \geq \frac{\nu+6}{10}$. If $\nu$ is sufficiently large, then $H$ is hamiltonian.

The main purpose of this chapter is to prove Conjecture 5.1.4. In fact, we proved a somewhat stronger result.

Theorem 5.1.5 If $H$ is 3-connected claw-free simple graph with $\nu \geq 196$, and if $\delta(H) \geq$ $\frac{\nu+5}{10}$, then either $H$ is hamiltonian, or $\delta(H)=\frac{\nu+5}{10}$ and $\mathrm{cl}(H)$ is the line graph of $G$ obtained from the Petersen graph $P_{10}$ by adding $\frac{\nu-15}{10}$ pendant edges at each vertex of $P_{10}$.

\subsection{Proof of Theorem 5.1.5}

The proof of Theorem 5.1.5 needs the following Theorem and Lemma. 
Theorem 5.2.1 (Chen, Lai, Li, Li and Mao, [13]) Let $G$ be a 3-edge-connected graph and let $S \subseteq V(G)$ be a vertex subset such that $|S| \leq 12$. Then either $G$ has a eulerian subgraph $C$ such that $S \subseteq V(C)$, or $G$ can be contracted to the Petersen graph in such a way that the preimage of each vertex of the Petersen graph contains at least one vertex in $S$.

Lemma 5.2.2 (Favaron and Fraisse, [15]) Let $S$ be a set of vertices of a graph $G$ contained in a eulerian subgraph of $G$ and let $C$ be a maximal eulerian subgraph of $G$ containing $S$. Assume that some component $A$ of $G-V(C)$ is not an isolated vertex and is related to $C$ by at least $r$ edges. Then

(i). $G$ contains a matching $T$ of $r+1$ edges such that at most $2 r$ edges of $G$ are adjacent to two distinct edges of $T$.

(ii). The number $m(G)$ of edges of $G$ is related to the minimum degree $\delta(H)$ of the line graph $H$ of $G$ by $m(G) \geq(r+1) \delta(H)-r+1$.

Portion of the proof of Theorem 5.1.5 (the treatment to deal with Claims 1 and 2) is a modification of Favaron and Fraisse's proof for Theorem 1 in [15], with Theorem 5.2.1 being utilized in our proof.

Proof of Theorem 5.1.5. By Theorem 5.1.1, the graph $H$ is hamiltonian if and only if its closure $c l(H)$ is hamiltonian. As $\nu(c l(H))=\nu(H), \delta(c l(H)) \geq \delta(H)$, and $c l(H)$ is 3-connected, the graph $c l(H)$ satisfies the same hypotheses as $H$. Hence it suffices to prove Theorem 5.1.5 for closed claw-free graphs.

By Theorem 5.1.1, we may assume that $H$ is the line graph of a triangle-free graph $G$ (i.e., $H=L(G)$ ), and suppose that $H$ is 3-connected and satisfies $\delta(H) \geq \frac{\nu(H)+5}{10}$. Assume by contradiction that neither of the conclusions of Theorem 5.1.5 holds. By Theorem 1.0.1, $G$ does not contain a dominating eulerian graph.

Let $B=\left\{v \in V(G) \mid d_{G}(v)=1,2\right\}$. Since $H$ is 3 -connected, the sum of degrees of the two ends of each edge in $G$ is at least 5 and thus the set $B$ is independent. Let $X_{0}=N_{G}(B)$. We name the vertices of $X_{0}$ as $x_{1}, x_{2}, \cdots, x_{p}$ in the following way. Assume the vertices $x_{1}, \cdots, x_{i}$ are already defined or else put $i=0$. Let $y_{i+1}$ denote 
a vertex of $B$ which is adjacent to some vertex of $X_{0}-\left\{x_{1}, \cdots, x_{i}\right\}$. Either $y_{i+1}$ has exactly one neighbor in $X_{0}-\left\{x_{1}, \cdots, x_{i}\right\}$ and we name it $x_{i+1}$, or $y_{i+1}$ has exactly two neighbors in $X_{0}-\left\{x_{1}, \cdots, x_{i}\right\}$ and we name them $x_{i+1}$ and $x_{i+2}$ and put $y_{i+2}=y_{i+1}$. Let $Y_{0}=\left\{y_{1}, \cdots, y_{p}\right\}$. We note that if $1 \leq i<j \leq p$, then $y_{i} y_{j} \notin E(G)$ and $y_{i} x_{j} \notin E(G)$, except for the edges $y_{i} x_{i+1}$ when $y_{i}=y_{i+1}$; and that the components of the subgraph induced by the edges $x_{i} y_{i}, 1 \leq i \leq p$, are paths of length 1 or 2 .

Consider now a matching $M$ of $G$ formed by $q-p$ edges $x_{i} y_{i}$ of $G, p+1 \leq i \leq q$, considered in this order and such that

(i) the sets $X_{0}, Y_{0}, X=\left\{x_{p+1}, \cdots, x_{q}\right\}$ and $Y=\left\{y_{p+1}, \cdots, y_{q}\right\}$ are pairwise disjoint,

(ii) for $p+1 \leq i<j \leq q, y_{i} y_{j}, y_{i} x_{j} \notin E(G)$.

We choose this matching as large as possible subject to the conditions (i) and (ii). Note that by the definition of $X_{0}$ and $Y_{0}$, the whole set $B$ is disjoint from $X \cup Y$ and that Property (ii) holds for any $i$ and $j$ with $1 \leq i<j \leq q$.

Let $J$ be the set of indices $j$ between $p+1$ and $q$ such that $y_{j}$ is adjacent to some vertex $z \notin X_{0} \cup Y_{0} \cup X \cup Y$ with $y_{k} z \notin E(G)$ for $1 \leq k<j$. For each $j \in J$ we choose such a vertex $z_{j}$ and we put $I=\{p+1, \cdots, q\}-J$. Let $X_{I}=\left\{x_{i} \in X \mid i \in I\right\}$, $X_{J}=\left\{x_{i} \in X \mid i \in J\right\}, Y_{I}=\left\{y_{i} \in Y \mid i \in I\right\}$ and $Y_{J}=\left\{y_{i} \in Y \mid i \in J\right\}$.

Claim 1. (Favaron and Fraisse, [15]) The set $S=X_{0} \cup X_{I} \cup Y_{J}$ is not contained in any eulerian subgraph of $G$.

Proof. Suppose Claim 1 is false and let $C$ be a maximal eulerian subgraph of $G$ containing $S=X_{0} \cup X_{I} \cup Y_{J}$ and $R=V(G)-V(C)$. By the assumption that $G$ has no dominating eulerian subgraphs, at least one component $A$ of $G[R]$ is not a single vertex. This component $A$ is disjoint from $Y_{0}$ since the vertices of $Y_{0}$ are isolated in $G[R]$.

Suppose first that every vertex of $A$ has a neighbor in $C$. Then, if $u v$ is an edge of $A$ and if $s$ denotes the number of edges between $A$ and $C, s \geq d_{C}(u)+d_{C}(v)+|A|-2$. Since $G$ is triangle-free, $d_{A}(u)+d_{A}(v) \leq|A|$ and thus $d_{G}(u)+d_{G}(v)=d_{C}(u)+d_{C}(v)+d_{A}(u)+$ $d_{A}(v) \leq d_{C}(u)+d_{C}(v)+|A|$. Hence $s \geq d_{G}(u)+d_{G}(v)-2 \geq \delta(H)$. Apply Lemma 5.2.2 with $r=\delta(H)$ to conclude that the number of edges of $G$ satisfies $m(G) \geq \delta^{2}(G)+1$. Since $\delta(H) \geq \frac{\nu(H)+5}{10}$, then $m(G)=\nu(H) \leq 10 \delta(H)-5$, and so $\delta^{2}(H)+1 \leq 10 \delta(H)-5$, 
contrary to the hypothesis that $\nu(H) \geq 196$.

Therefore $A$ contains a vertex $z$ such that $N_{G}(z) \subseteq A$. Then $z \notin X_{0} \cup Y_{0} \cup X \cup Y$ and the neighbors of $z$ are all in $Y_{I} \cup X_{J} \cup\left(R-\left(Y_{0} \cup Y_{I} \cup X_{J}\right)\right)$.

If $z$ has a neighbor in $Y_{I}$, let $i$ be the least index such that $y_{i} \in Y_{i}$ and $z y_{i} \in E(G)$. Since $z$ has no neighbor in $Y_{J}, z y_{k} \notin E(G)$ for all $k<i$, in contradiction to the definition of $I$. Hence $z$ has no neighbor in $Y_{I}$, and thus in $Y$.

If $z$ has a neighbor in $X_{J}$, let $x_{j}$ be the vertex of $N_{G}(z) \cap X_{J}$ with the largest index. Consider the ordered sets $X^{\prime}=\left\{x_{p+1}, \cdots, x_{j-1}, x_{j}, z_{j}, x_{j+1}, \cdots, x_{q}\right\}$ and $Y^{\prime}=$ $\left\{y_{p+1}, \cdots, y_{j-1}, z, y_{j}, y_{j+1}, \cdots, y_{q}\right\}$. Then vertex $z$ is neither adjacent to any $x_{k}$ with $k>j$ by the definition of $x_{j}$ and since $z$ has no neighbor in $X_{I}$, nor to any vertex of $Y$ as said above. The vertex $z_{j}$ is not adjacent to any vertex $y_{k}$ with $k<j$ by the choice of $z_{j}$. If $z z_{j} \notin E(G)$, then the sets $X^{\prime}$ and $Y^{\prime}$ define a matching $M^{\prime}$ which satisfies (i) and (ii), and thus which contradicts the maximality of $M$. If $z z_{j} \in E(G)$, then the eulerian subgraph $G\left[\left(E(C)-E\left(C^{\prime}\right)\right) \cup\left(E\left(C^{\prime}\right)-E(C)\right)\right]$, with $C^{\prime}=y_{j} z_{j} z x_{j} y_{j}$, satisfies $V(C) \cap V\left(C^{\prime}\right)=\left\{y_{j}\right\}$ since $z$ has no neighbor in $C$, and thus contradicts the maximality of $C$. Hence $N_{G}(z) \cap X_{J}=\emptyset$ and $z$ has no neighbor in $X$.

Finally if $z$ has a neighbor $t$ in $R-\left(Y_{0} \cup Y_{I} \cup X_{J}\right)$, then the matching $M^{\prime \prime}$ corresponding to the ordered sets $X^{\prime \prime}=\left\{t, x_{p+1}, \cdots, x_{q}\right\}$ and $Y^{\prime \prime}=\left\{z, y_{p+1}, \cdots, y_{q}\right\}$ satisfies the conditions (i) and (ii) since $z$ has no neighbor in $X \cup Y$. This contradicts the maximality of $M$ and achieves the proof of Claim 1 .

Claim 2. (Favaron and Fraisse, [15]) G must be contractible to the Petersen graph.

Proof. By contradiction. Suppose that $G$ can not be contracted to the Petersen graph. Let $G^{1}$ be the graph or multigraph obtained from $G$ by deleting the vertices of degree 1 or 2 and replacing each path $a y b$ where $d_{G}(y)=2$ by the edge $a b$. Since $G$ is essentially 3-edge-connected, $G^{1}$ is 3-edge-connected. Moreover, to each eulerian subgraph $C$ of $G^{1}$ corresponds a eulerian subgraph of $G$ containing $V(C)$. Since $S \cap B=\emptyset$, the set $S$ is contained in $V\left(G^{1}\right)$. Since $S$ is not contained in any eulerian subgraph of $G$ by Claim $1, S$ is not contained in any eulerian subgraph of $G^{1}$. By Theorem 5.2.1, $|S| \geq 13$. Let $F=\left\{x_{i} y_{i} \mid 1 \leq i \leq 13\right\}, P=\left\{x_{i} \mid 1 \leq i \leq 13\right\}$ and $Q=\left\{y_{i} \mid 1 \leq i \leq 13\right\}$. We suppose that 
$F$ consists of $l$ paths of length 2 with $0 \leq l \leq 6$ and $13-2 l$ edges of a matching. Then $|P|=13$ and $|Q|=13-l$. We know that $Q$ is independent, that $y_{i} x_{j} \notin E(G)-F$ for any $y_{i} \in Q$ and $x_{j} \in P$ with $1 \leq i<j \leq 13$, and that $G$ is triangle-free. Hence, two different edges of $F$ are joined by at most one edge of $G$ which is of type $x_{i} x_{j}$ or $x_{i} y_{j}$ with $1 \leq i<j \leq 13$. More precisely, we can give an upper bound on the number $\mu$ of edges of $G$ which are adjacent to two different edges of $F$. For a given value of $l$, this number can be maximum if the $l$ paths of $F$ occur with smaller indices than those of the $13-2 l$ edges of the matching. This is due to the fact that the $l$ vertices $y_{i}$ belonging to paths of length 2 have degree 2 and thus they cannot be adjacent by an edge not in $F$ to any vertex $x_{i}$ with $i<j$. When this condition is fulfilled, there are at most $l^{2}$ edges between the vertices $x_{1}, x_{2}, \cdots, x_{2 l}$ (since the number of edges of a trianglefree graph of order $2 l$ is at most $\left.(2 l)^{2} / 4\right), 2 l(13-2 l)$ edges of type $x_{i} y_{j}$ between the sets $\left\{x_{1}, x_{2}, \cdots, x_{2 l}\right\}$ and $\left\{y_{2 l+1}, y_{2 l+2}, \cdots, y_{13}\right\}$, and $\frac{(13-2 l)(13-2 l-1)}{2}$ edges of type $x_{i} x_{j}$ or $x_{i} y_{j}$ with $i<j$ between the vertices of the set $\left\{x_{2 l+1}, \cdots, x_{13}, y_{2 l+1}, \cdots, y_{13}\right\}$. Then $\mu \leq$ $l^{2}+2 l(13-2 l)+\frac{(13-2 l)(13-2 l-1)}{2}=l-l^{2}+78$. Counting the edges of $G-F$ adjacent to some edge of $F$, we find at least $(13-2 l) \delta(H)$ edges adjacent to an edge of a matching of $F$ and $2 l(\delta(H)-1)$ edges adjacent to an edge of a path of length 2 (since each vertex $y_{i}$ on such a path has degree 2 in $G$ ). At most $l-l^{2}+78$ of these edges have their two endvertices in $P \cup Q$ and are thus counted twice. Hence $m(G) \geq(13-2 l) \delta(H)+2 l(\delta(H)-1)-\left(l-l^{2}+78\right)+13$, that is $\nu(H)=m(G) \geq 13 \delta(H)+l^{2}-3 l-65 \geq 13 \delta(H)-67 \geq 10 \delta(H)-4$ since $l$ is an integer between 0 and 6 and $\nu(H) \geq 196$. This contradicts to the hypothesis that $\delta(H) \geq \frac{\nu(H)+5}{10}$, and so Claim 2 must hold.

By Claim 2, $G$ can be contracted to the Petersen graph $P_{10}$. Let $v_{1}, v_{2}, \cdots, v_{10}$ be the ten vertices of the Petersen graph $P_{10}$, and $W_{i}$ be the preimage of $v_{i}(i=1,2, \cdots, 10)$. Denote $\mathcal{S} \mathcal{V}=\left\{v \in V(G) \mid d_{G}(v) \geq 12\right\}$. Since $d_{G}(u)+d_{G}(v)-2 \geq \delta(H) \geq 21$ for every edge $e=u v \in E(G)$, we have either $d_{G}(u) \geq 12$ or $d_{G}(v) \geq 12$. So we have

$$
\text { for every edge } e=u v \in E(G) \text {, either } u \in \mathcal{S} \mathcal{V} \text { or } v \in \mathcal{S} \mathcal{V} \text {. }
$$

Moreover, if $u, v \notin \mathcal{S V}$, then $u v \notin E(G)$. By the hypothesis of Theorem 5.1.5 that $H$ is 3-connected, we have

$G$ is essentially 3-edge-connected. 
Let $W \in\left\{W_{i} \mid 1 \leq i \leq 10\right\}$. Note that $G$ is contracted to $P_{10}$. Then $\mid N_{W}(V(G)-$ $V(W)) \mid=3$. If for any two vertices $w_{1}, w_{2} \in N_{W}(V(G)-V(W))$, there is a dominating $\left(w_{1}, w_{2}\right)$-trail in $W$, then say $W$ is dominatable.

Claim 3. Let $W^{\prime}$ be a graph obtained from $W$ by deleting the vertices of degree 1 . If $E\left(W^{\prime}\right) \neq \emptyset$, then $W^{\prime}$ is 2-edge-connected. Therefore $W^{\prime}$ contains some cycle.

Proof. Since $G$ is contracted to the $P_{10}$ and $W$ is the preimage of some vertex $v_{i}$, we may assume that $[V(W), V(G)-V(W)]_{G}=\left\{e_{1}, e_{2}, e_{3}\right\}$, where $e_{1}, e_{2}, e_{3}$ are edges adjacent to $v_{i}$ in $P_{10}$. Suppose that $W^{\prime}$ contains a cut-edge $e=z_{1} z_{2}$. Then $e$ is also a cut-edge of $W$. Let $\left(U_{1}, V_{1}\right)$ be the partition of $V(W)$ such that $\left[U_{1}, V_{1}\right]_{W}=\{e\}$ and $z_{1} \in U_{1}$ and $z_{2} \in V_{1}$. Since $z_{1}, z_{2} \in V\left(W^{\prime}\right)$, we have $d_{W}\left(z_{1}\right) \geq 2$ and $d_{W}\left(z_{2}\right) \geq 2$. Thus $E\left(G\left[U_{1}\right]\right) \neq \emptyset$ and $E\left(G\left[V_{1}\right]\right) \neq \emptyset$. Note that $[V(W), V(G)-V(W)]_{G}=\left\{e_{1}, e_{2}, e_{3}\right\}$. We may assume that the number of edge joining $U_{1}$ and $V(G)-V(W)$ is 1 , say $e_{1}$. Then $\left\{e_{1}, e\right\}$ is an essential edge-cut in $G$, contrary to (5.2). So Claim 3 holds.

Claim 4. If $\alpha^{\prime}(W)=1$, then $W=K_{1, p}$ for some $p \geq 1$. Therefore all three edges in $[V(W), V(G)-V(W)]_{G}$ must be incident with the vertex of $K_{1, p}$ with degree $p$, and so $H_{1}$ is dominatable.

Proof. Since $W$ is a connected triangle-free graph and $\alpha^{\prime}(W)=1, G$ is acyclic. By Claim 3 and $\alpha^{\prime}(W)=1, W=K_{1, p}$ for some $p \geq 1$.

Claim 5. Suppose that $\alpha^{\prime}(W)=t \in\{2,3, \cdots, 5\}$ and $\left\{u_{1} a_{1}, u_{2} a_{2}, \cdots, u_{t} a_{t}\right\}$ is a matching in $W$. Suppose that $u_{i} \in \mathcal{S} \mathcal{V}(i=1,2, \cdots, t)$. Then $V(W) \cap \mathcal{S} \mathcal{V}=\left\{u_{1}, u_{2}, \cdots, u_{t}\right\}$ and $E\left(W-\left\{u_{1}, u_{2}, \cdots, u_{t}\right\}\right)=\emptyset$.

Proof. Let $A=\left\{u_{1}, \cdots, u_{t}, a_{1}, \cdots, a_{t}\right\}, A_{1}=A-u_{i}$ and $A_{2}=A-a_{i}$. By $\alpha^{\prime}(W)=t$, $E(W-A)=\emptyset$. Note that $G$ is triangle-free and $\mathcal{S} \mathcal{V}=\left\{v \in V(G) \mid d_{G}(v) \geq 12\right\}$. For each $z \in V(W)-A, d_{W}(z) \leq 5$ and so $d_{G}(z) \leq 8$. Thus $z \notin \mathcal{S} \mathcal{V}$.

Since $G$ does not contain a triangle and $\alpha^{\prime}(W)=t \leq 5$, by $d_{G}\left(u_{i}\right) \geq 12$, we have $N_{W}\left(u_{i}\right)-A_{1} \neq \emptyset$. Thus $N_{W}\left(a_{i}\right) \subseteq A_{2}$ (otherwise, $\left\{u_{1} a_{1}, \cdots, u_{i-1} a_{i-1}, u_{i+1} a_{i+1}, \cdots, u_{t} a_{t}\right.$, $\left.u_{i} u, a_{i} a\right\}$ is a matching of $W$, where $u \in N_{W}\left(u_{i}\right)-A_{1}$ and $a \in N_{W}\left(a_{i}\right)-A_{2}$, contrary to the assumption that $\alpha^{\prime}(W)=t$ ). Since $G$ is triangle-free, we have $d_{W}\left(a_{i}\right) \leq 5$, 
and so $d_{G}\left(a_{i}\right) \leq 8$. Thus $a_{i} \notin \mathcal{S} \mathcal{V}$. Therefore $\mathcal{S} \mathcal{V} \cap V(W)=\left\{u_{1}, u_{2}, \cdots, u_{t}\right\}$, and $E\left(W-\left\{u_{1}, u_{2}, \cdots, u_{t}\right\}\right)=\emptyset$.

Claim 6. If $\alpha^{\prime}(W)=t \in\{2,3,4\}$, then $W$ is dominatable.

Proof. Suppose that $\alpha^{\prime}(W)=t$ and $\left\{u_{1} a_{1}, \cdots, u_{t} a_{t}\right\}$ is a matching in $W$. Without loss of generality, we assume that $u_{i} \in \mathcal{S} \mathcal{V}(i=1,2, \cdots, t)$ by $(5.1)$. By Claim $5, \mathcal{S} \mathcal{V} \cap V(W)=$ $\left\{u_{1}, u_{2}, \cdots, u_{t}\right\}$, and $E\left(W-\left\{u_{1}, u_{2}, \cdots, u_{t}\right\}\right)=\emptyset$. Let $w_{1}, w_{2}, w_{3} \in N_{W}(V(G)-V(W))$ and $w_{1} z_{1}, w_{2} z_{2}, z_{3} w_{3} \in[V(W), V(G)-V(W)]_{G}$. If $w_{1}=w_{2}$ and $d_{W}\left(w_{1}\right)=1$, then $\left\{z_{3} w_{3}, w_{1} x\right\}$ is an essential edge-cut in $G$ for some $x \in N_{W}\left(w_{1}\right)$, contrary to (5.2). So we have $d_{W}\left(w_{1}\right) \geq 2$ if $w_{1}=w_{2}$.

Suppose, by contradiction, that $W$ does not exist a dominating $\left(w_{1}, w_{2}\right)$-trail. If $w_{1} \neq w_{2}$, we let $K_{1}=W+\left\{w_{1} w, w_{2} w\right\}$ where $w$ is a new vertex; if $w_{1}=w_{2}$, we let $K_{1}=W$ and $w=w_{1}$. Let $K=K_{1}-D_{1}\left(K_{1}\right)$. Then $u_{1}, \cdots, u_{t}, w \in V(K)$, and $K$ is 2-edge-connected by Claim 3. Let $S=\left\{u_{1}, \cdots, u_{t}\right\} \cup\{w\}$. Then $K-S$ is edgeless, and $K$ does not have an $S$-eulerian subgraph. By Theorem 2.0.13, $K$ is contractible to a member $L \in \mathcal{F}$ ( see Figures $2.4,2.5,2.6$ ) such that $S$ intersects the preimage of every vertex in $B(L)$. Note that for each $L \in \mathcal{F}, d_{L}\left(b_{i}\right)=2(i=1,2,3)$ and the set of degree 2 vertices is independent. Without loss of generality, we assume that the preiamges of $b_{1}, b_{2}$ do not contain $w$.

Note that $[V(W), V(G)-V(W)]_{G}=\left\{w_{1} z_{1}, w_{2} z_{2}, z_{3} w_{3}\right\}$. Suppose that $w \in V(L)$. Then $w_{1}, w_{2} \in V(L)$. If $w_{1} \neq w_{2}$, then $d_{L}(w)=2$. Thus $w_{1}, w_{2} \notin\left\{b_{1}, b_{2}\right\}$. If $w_{1}=w_{2}$, then $w_{1}=w_{2}=w$. Thus $w_{1}, w_{2} \notin\left\{b_{1}, b_{2}\right\}$ still hold. Since either $w_{3} \notin V\left(P I\left(b_{1}\right)\right)$ or $w_{3} \notin V\left(P I\left(b_{2}\right)\right)$, we may assume that $w_{3} \notin V\left(P I\left(b_{1}\right)\right)$. Thus $\left[V\left(P I\left(b_{1}\right)\right), V(G)-\right.$ $V(W)]=\emptyset$ and the set of two edges adjacent to $V\left(P I\left(b_{1}\right)\right)$ is an essential edge-cut of $G$, contrary to (5.2). So $w \notin V(L)$. We assume that the preimage of some $b_{i}\left(\notin\left\{b_{1}, b_{2}\right\}\right)$ contains $w$. Thus $w_{1}, w_{2} \notin V\left(P I\left(b_{i}\right)\right)(i=1,2)$. Therefore either $\mid\left[V\left(P I\left(b_{1}\right)\right), V(G)-\right.$ $V(W)] \mid=0$ or $\left|\left[V\left(P I\left(b_{2}\right)\right), V(G)-V(W)\right]\right|=0$. Without loss of generality, we assume that $\left|\left[V\left(P I\left(b_{1}\right)\right), V(G)-V(W)\right]\right|=0$. Then the set of two edges adjacent to $V\left(P I\left(b_{1}\right)\right)$ is an essential edge-cut of $G$, contrary to (5.2).

Claim 7. If $\alpha^{\prime}(W)=t \geq 1$, then $|E(W)| \geq t \delta(H)+2 t-t^{2}-3$. 
Proof. Let $\left\{u_{1} v_{1}, \cdots, u_{t} v_{t}\right\}$ be a matching in $W$. Then $E\left(W-\left\{u_{1}, \cdots, u_{t}, v_{1}, \cdots, v_{t}\right\}\right)=$ $\emptyset$, and for any pair of $u_{i} v_{i}, u_{j} v_{j}(i \neq j),\left|\left[\left\{u_{i}, v_{i}\right\},\left\{u_{j}, v_{j}\right\}\right]_{W}\right| \leq 2$ since $W$ does not contain a triangle. Since for $\sum_{v \in V(W)} d_{W}(v)$, the edges of $u_{i} v_{i}$ and the edges in $\left[\left\{u_{i}, v_{i}\right\},\left\{u_{j}, v_{j}\right\}\right]_{W}$ are counted twice, and since $\left|[V(W), V(G)-V(W)]_{G}\right|=3$, we have $|E(W)|=\sum_{v \in V(W)} d_{W}(v)-$ $\left|\left\{u_{1} v_{1}, u_{2} v_{2}, \cdots, u_{t} v_{t}\right\}\right|-\sum_{i \neq j}\left|\left[\left\{u_{i}, v_{i}\right\},\left\{u_{j}, v_{j}\right\}\right]_{W}\right| \geq\left(\sum_{v \in V(W)} d_{G}(v)-3\right)-t-2\left(\begin{array}{c}t \\ 2\end{array}\right)$. Since $\delta(H) \leq d_{G}\left(u_{i}\right)+d_{G}\left(v_{i}\right)-2$ for each $u_{i} v_{i}$, we have $|E(W)| \geq t(\delta(H)+2)-3-t-2\left(\begin{array}{c}t \\ 2\end{array}\right)=$ $t \delta(H)+2 t-t^{2}-3$.

Now we finish the proof of Theorem 5.1.5. Let $\mid\left\{v_{i} \mid v_{i}\right.$ is a trivial vertex in $\left.P_{10}\right\} \mid=s$. By (5.1), the set of all trivial vertices in $P_{10}$ is independent. Since $\alpha^{\prime}\left(P_{10}\right)=4$, we have $0 \leq s \leq 4$. If $s=0$, then each $v_{i}$ is nontrivial vertex. Thus $\left|E\left(W_{i}\right)\right| \geq \delta(H)-2$ by Claim 7. Therefore $m(G)=\sum_{i=1}^{10}\left|E\left(W_{i}\right)\right|+15 \geq 10(\delta(H)-2)+15=10 \delta(H)-5$. By the hypothesis of Theorem 5.1.5, we have $\delta(H)=\frac{\nu(H)+5}{10},\left|E\left(W_{i}\right)\right|=\delta(H)-2, \alpha^{\prime}\left(W_{i}\right)=1$ and $W_{i}=K_{1, p}$, where $p=\delta(H)-2=\frac{\nu(H)-15}{10}$.

If $s \geq 1$, without loss of generality, we assume that $v_{1}$ is trivial. Since $P_{10}-v_{1}$ has a spanning cycle, there exists a $W_{i}$, say $W_{10}$, such that $\alpha^{\prime}\left(W_{10}\right) \geq 5$ by Claims 4 and 6 . If $s \leq 3$, then $m(G)=\sum_{i=1}^{10}\left|E\left(W_{i}\right)\right|+15 \geq(10-s-1)(\delta(H)-2)+(5 \delta(H)-18)+15 \geq$ $6(\delta(H)-2)+5 \delta(H)-3=11 \delta(H)-15 \geq 10 \delta(H)-4$. Thus $\delta(H) \leq \frac{\nu(H)+4}{10}$, a contradiction. So $s=4$. By Claims 3,6 and $\delta(H) \geq \frac{\nu(H)+5}{10}, \alpha^{\prime}\left(W_{10)}\right)=5$. If there exists some $W_{j}(j \neq 10)$ such that $\alpha^{\prime}\left(W_{j}\right) \geq 2$, then $m(G)=\sum_{i=1}^{10}\left|E\left(W_{i}\right)\right|+15 \geq\left|E\left(W_{10}\right)\right|+\left|E\left(W_{j}\right)\right|+$ $4(\delta(H)-2)+15=(5 \delta(H)-18)+(2 \delta(H)-3)+4 \delta(H)+7=11 \delta(H)-17 \geq 10 \delta(H)+4$, a contradiction. So the number of $W_{i}$ with $\alpha^{\prime}\left(W_{i}\right)=1$ is 5 . Without loss of generality, we assume that $\alpha^{\prime}\left(W_{i}\right)=1(i=5,6,7,8,9)$ and $\alpha^{\prime}\left(W_{10}\right)=5$. Let $\left\{e_{1} f_{1}, e_{2} f_{2}, e_{3} f_{3}, e_{4} f_{4}, e_{5} f_{5}\right\}$ be a matching of $W_{10}$ and $B=\left\{e_{1}, \cdots, e_{5}, f_{1}, \cdots, f_{5}\right\}$ and $Z=W_{10}[B]$. By (2), we assume that $e_{i} \in \mathcal{S} \mathcal{V}(i=1,2, \cdots, 5)$. By Claim $5, \mathcal{S} \mathcal{V} \cap V\left(W_{10}\right)=\left\{e_{1}, e_{2}, \cdots, e_{5}\right\}$, and $E\left(W_{10}-\left\{e_{1}, e_{2}, \cdots, e_{5}\right\}\right)=\emptyset$. 
If $|E(Z)| \leq 16$, then $\left|E\left(W_{10}\right)\right|=\sum_{v \in B} d_{G}(v)-|E(Z)|-3 \geq 5(\delta(H)+2)-16-3=$ $5 \delta(H)-9$. Thus $m(G)=\sum_{i=5}^{9}\left|E\left(W_{i}\right)\right|+\left|E\left(W_{10}\right)\right|+15 \geq 5(\delta(H)-2)+(5 \delta(H)-9)+15=$ $10 \delta(H)-4$, and so $\delta(H) \leq \frac{\nu(H)+4}{10}$, a contradiction. So we have

$$
|E(Z)| \geq 17
$$

If $Z$ is collapsible, then $W_{10}-D_{1}\left(W_{10}\right)$ is collapsible by Theorem 2.0.7(iii). Thus for any pair of vertices $u, v \in W_{10}-D_{1}\left(W_{10}\right), W_{10}-D_{1}\left(W_{10}\right)$ has a spanning $(u, v)$-trail by Lemma 2.0.12. Then for any pair of vertices $u, v \in V\left(W_{10}\right), W_{10}$ has a dominating $(u, v)$-trail, and so $W_{10}$ is dominatable. Since each $W_{i}(i=1,2,3,4)$ is a trivial graph, since each $W_{i}(i=5,6, \cdots, 9)$ is dominatable, and since $P_{10}-v_{1}$ has a spanning cycle, $G$ has a dominating eulerian subgraph, a contradiction. So $Z$ is not collapsible. Moreover,

$$
W_{10}-D_{1}\left(W_{10}\right) \text { is not collapsible. }
$$

Therefore $Z$ is not 2-edge-connected by Lemma 2.0.15.

Let $K \subseteq Z$ with $|V(K)|=8$. Suppose that $|E(K)| \geq 14$. Then $K$ is 2-edge-connected by Lemma 2.0.14. If $\left|D_{2}(K)\right| \geq 2$, then $|E(K)| \leq 2+2+9=13$ by Lemma 2.0.14(iv), a contradiction. So $\left|D_{2}(K)\right| \leq 1$. By Lemma 2.0 .11 and by the fact that $G$ is trianglefree, $K$ is collapsible. By Claim 3 and Theorem 2.0.7(iii), $M_{10}-D_{1}\left(M_{10}\right)$ is collapsible, contrary to (5.4). So

$$
|E(K)| \leq 13
$$

Suppose that $Z$ is not connected and $Z_{1}$ is a component of $Z$. Then $\left|V\left(Z_{1}\right)\right| \in$ $\{2,4,6,8\}$. By Lemmas 2.0.14(ii),(iv) and (5.3), $\left|V\left(Z_{1}\right)\right|$ is either 2 or 8. We may assume that $\left|V\left(Z_{1}\right)\right|=2$ and $Z_{2}=Z-V\left(Z_{1}\right)$. Then $\left|E\left(Z_{1}\right)\right|=1,\left|V\left(Z_{2}\right)\right|=8$ and $\left|E\left(Z_{2}\right)\right| \geq 16$, contrary to (5.5). So $Z$ is connected. Let $X$ be a cut-edge of $Z$ and $Z_{3}, Z_{4}$ are components of $Z-X$ with $\left|V\left(Z_{3}\right)\right| \leq\left|V\left(Z_{4}\right)\right|$. By Lemma 2.0.14 and (5.3), $\left|V\left(Z_{3}\right)\right|$ is either 1 or 2. If $\left|V\left(Z_{3}\right)\right|=2$, then $\left|E\left(Z_{4}\right)\right| \geq 17-2=15$, contrary to $(5.5)$. So $\left|V\left(Z_{3}\right)\right|=1,\left|V\left(Z_{4}\right)\right|=9$, $\left|\left[V\left(Z_{3}\right), V\left(Z_{4}\right)\right]_{Z}\right|=1$ and $\left|E\left(Z_{4}\right)\right| \geq 16$.

By (5.5) and Lemma 2.0.14, $Z_{4}$ is 3-edge-connected. Let $Z_{4}^{\prime}$ be the reduction of $Z_{4}$. Then $Z_{4}^{\prime}$ is still 3-edge-connected and $\left|V\left(Z_{4}^{\prime}\right)\right| \leq 9$. Thus $Z_{4}^{\prime}=K_{1}$ by Theorem 2.0.11, 
that is, $Z_{4}$ is collpasible. By Claim 3 and Theorem 2.0.7(iii), $W_{10}-D_{1}\left(W_{10}\right)$ is collapsible, contrary to (5.4). 


\section{Bibliography}

[1] J. A. Bondy and U. S. R. Murty, "Graph Theory with Applications". American Elsevier (1976).

[2] H. J. Broersma and L. M. Xiong, A note on minimum degree conditions for supereulerian graphs, Discrete Applied Math, to appear.

[3] X. T. Cai, Connected Eulerian spanning subgraphs, Chinese Quarterly J. of Mathematics, 5 (1990) 78-84.

[4] P. A. Catlin, A reduction method to find spanning Eulerian subgraphs, J. Graph Theory, 12 (1988) 29-44.

[5] P. A. Catlin, Double cycle covers and the Petersen graph, J. Graph Theory, 13 (1989) 465-483.

[6] P. A. Catlin, Supereulerian graph, collapsible graphs and 4-cycles, Congressus Numerantium, 56 (1987) 223-246.

[7] P. A. Catlin and Z. H. Chen, Nonsupereulerian graphs with large size, "Graph Theory, Combinatorics, Algorithms and Applications", eds. by Y. Alavi, F. R. K. Chung, R. L. Graham and D. F. Hsu, SIAM (1991) 83-95.

[8] P. A. Catlin, Z. Han and H.-J. Lai, Graphs without spanning closed trails, Discrete Math. 160 (1996) 81-91.

[9] P. A. Catlin and X. W. Li, Supereulerian graphs of minimum degree at least 4, J. Advances in Mathematics 28(1999), 65-69. 
[10] Z. H. Chen, Supereulerian graphs and the Petersen graph, J. Combinat. Math. Comb. Comput. 9(1991), 79-89.

[11] Z. H. Chen, On extremal non supereulerian graph with clique number $m$, Ars Combinatoria, 36 (1993) 161-169.

[12] Z. H. Chen and H.-J. Lai, Supereulerian graphs and the Petersen graph, II, ARS Combinatoria 48(1998), 271-282.

[13] Z.-H. Chen, H.-J. Lai, X. Li, D. Li and J. Mao, Eulerian subgraphs in 3-edge-connected graphs and hamiltonian line graphs, J. Graph Theory, accepted.

[14] O. Favaron, E. Flandrin, H. Li and Z. Ryjáček, Cliques covering and degree conditions for hamiltonicity in claw-free graphs, Discrete Math., in press.

[15] Odile Favaron and P. Fraisse, Hamiltonicity and minimum degree in 3-connected claw-free graphs, J. Combin. Theory Ser. B, 82(2001), 297-305.

[16] F. Harary and C. St. J. A. Nash-Williams, On eulerian and hamiltonian graphs and line graphs, Canad. Math. Bull. 8(1965), 701-709.

[17] F. Jaeger, A note on subeulerian graphs, J. Graph Theory, 3 (1979) 91-93.

[18] E. J. Kuipers and H. J. Veldman, Recognizing claw-free hamiltonian graphs with large minimum degree, Memorandum 1437, University of Twente, 1998, submitted for publication.

[19] H.-J. Lai, Graphs whose edges are in small cycles, Discrete Math. 94 (1991) 11-22.

[20] H.-J. Lai, Eulerian subgraphs containing given vertices and hamiltonian line graphs, Discrete Math. 178 (1998) 93-107.

[21] C. St. J. A. Nash-Williams, Edge-disjoint spanning trees of finite graphs, J. London Math Soc. 36 (1961) 445-450.

[22] M. M. Matthews and D. P. Sumner, Hamiltonian results in $K_{1,3}$-free graphs, J. Graph Theory, 8( 1984) 139-146. 
[23] P. Paulraja, On graphs admitting spanning Eulerian subgraphs, Ars Combinatoria, 24 (1987) 57-65.

[24] P. Paulraja, Research Problem 85, Discrete Math. 64 (1987) 109.

[25] Z. Ryjáček, On a closure concept in claw-free graphs, J. Combin. Theory Ser. B, 70(1997), 217-224.

[26] W. T. Tutte, On the problem of decomposing a graph into $n$ connected factors, J. London Math Soc. 36 (1961) 80-91.

[27] S. Zhan, Hamiltonian connectedness of line graphs, Ars Combinatoria, 22(1986) 8995. 